\title{
FORCED VIBRATION ANALYSIS OF A MULTIDEGREE IMPACT VIBRATOR
}

\author{
D. Pun, S . L . L au, S . S . L aW And D. Q. CaO \\ Department of Civil and Structural Engineering, Hong Kong Polytechnic University, \\ Hung Hom, Kowloon, Hong Kong
}

\begin{abstract}
The dynamics of a multidegree impact vibrator subject to harmonic loading is investigated. The system is represented by a lumped mass model which hits and rebounds from a rigid wall during vibration. The periodic solution to the equations of motion with $N$ forcing cycles and $P$ impacts is formulated. The variational equations and the resulting transition matrix for investigating local stability of the periodic solutions are derived. A two-degree-of-freedom example is analysed, and a variety of motion types are found. Chaotic windows are present between regions of periodic response, and at these boundaries $N$-P motions are prevalent. Low velocity impacts are evident at exciting frequencies away from the natural frequencies. Two basins of attraction are computed, and the sensitivity to initial conditions is noted. The quality of the $N-P$ motion is discussed from an engineering application perspective.
\end{abstract}

\section{INTRODUCTION}

The vibration of a system subject to displacement restraint is examined. The analysis of impacting systems has been of considerable interest to researchers, for there are many situations in engineering practice where such behaviour occurs. Some examples are cutting tools, jack hammers, compacting equipment, mechanical components which overshoot their designed travel and impact other parts under abnormal conditions. Shaw and Shaw [1] analysed the chaotic behaviour of an oscillator with an inverted impacting pendulum and considered motions with one and two impacts per response cycle. Thompson and Ghaffari [2] observed a cascade of period-doubling bifurcations leading to chaos for an impact oscillator in a study of mooring line dynamics. Shaw and Holmes [3] considered a piecewise unsymmetric oscillator which could be used to represent an impact vibrator. For this case they computed analytically period $N$ orbits with one impact per response cycle and examined their stability. Popplewell et al. [4] formulated the equations for the solution of period $N$ motion with $P$ number of impacts per response cycle ( $N-P$ motion) for a single-degree-of-freedom (DOF) impact damper. They presented numerical results for 2-1 to 5-1 motions, and performed experiments to verify their results. They further suggested that the oscillator should be excited at or slightly above its natural frequency for the damper to work best, and that the driving speed should be carefully controlled because response sensitivity is high in this region.

Masri [5] analysed a multidegree system with an impact damper which can be attached to any location on the system. He assumed damper motion of two symmetric impacts per 
load cycle, and obtained good correlations with corresponding experiments. Applications of the analysis to tall buildings demonstrated that the damper can be effective over a wide frequency range. Aidanpaa and Gupta [6] analysed in detail the one-impact motion of a two-DOF impact vibrator. They studied the influences of system parameters and noted behavioral similarities between one- and two-DOF systems. Nigm and Shabana [7] studied the dynamics of a multidegree system with an impact damper placed at one end. The damper is connected to the main system by a viscous damper and a spring. They analysed motion with two unsymmetric impacts per load cycle, and obtained experimental results that generally correlated with computations. Their experiments showed that there are frequency regimes for which steady state solutions apparently do not exist. The motion is such that for some time durations no impact takes place and the vibration amplitude builds up until collisions occur, and the amplitude decreases again until impact no longer materializes, and so on. Moore et al. [8] pointed out in their work on impact dampers in a cryogenic environment that amplitude and frequency of impact per forcing cycle are useful and important characterizations, and noted in their simulation work similar unsteady behaviour as observed in reference [7].

$\mathrm{Cao}$ and Shu [9] investigated a multidegree impact vibrator for $N-1$ motion and obtained robust stability bounds. This paper presents a solution method for periodic $N-P$ motion of a multi-DOF impact vibrator, which has not been analysed before. Such motion is significant because it includes the class of response in which impacts occur occasionally, and may be part of the apparently unsteady behaviour reported in references $[7,8]$. The transition matrix for the stability analysis of the periodic orbits is obtained through the perturbed motion technique. A two-DOF example is presented, for which regimes of $N-P$ motion and chaotic dynamics are found. The results are discussed with the aid of frequency spectra, phase diagrams, Poincaré maps, and basins of attraction. Finally, some comments with respect to the performance of impact machinery are made.

\section{FORMULATION}

\subsection{EQUATIONS OF MOTION}

Consider a multiple-DOF lumped mass system which experiences impact when the displacement $X_{1}$ of mass $M_{1}$ reaches the barrier at $B$, as shown in Figure 1. The following normalized quantities are introduced:

$$
\begin{gathered}
m_{i}=M_{i} / M_{1}, \quad k_{i}=K_{i} / K_{1}, \quad \xi_{i}=C_{i} /\left(2 \sqrt{\left.K_{1} M_{1}\right)},\right. \\
\omega=\Omega / \sqrt{K_{1} / M_{1}}, \quad t=T \sqrt{K_{1} / M_{1}},
\end{gathered}
$$

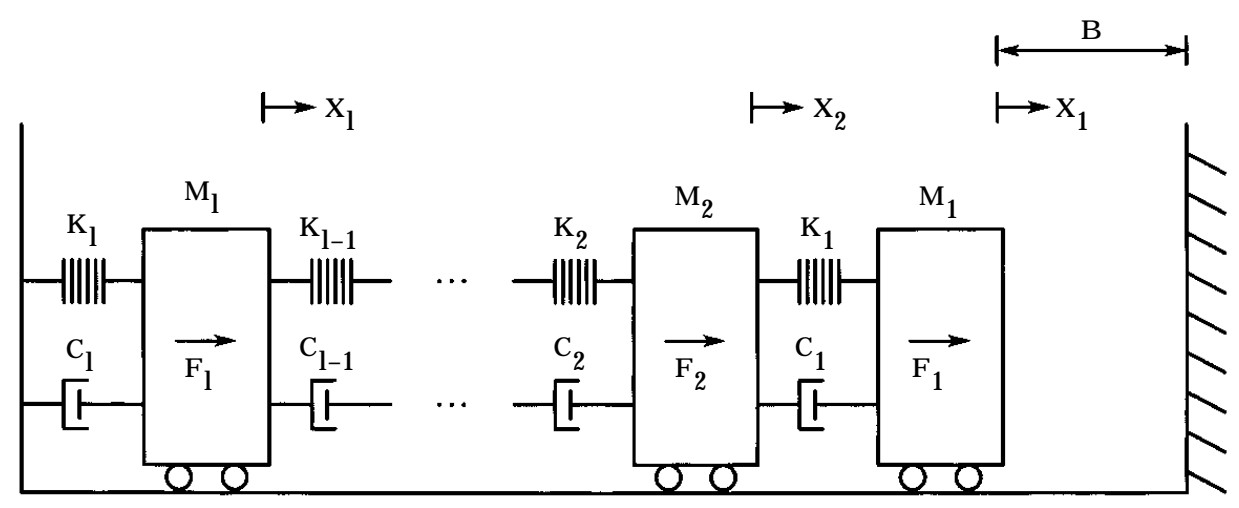

Figure 1. Impact vibrator model. 


$$
\begin{gathered}
F_{0}=\left(\sum_{i=1} F_{i}^{2}\right)^{1 / 2}, \quad f_{i}=F_{i} / F_{0}, \\
b=B K_{1} / F_{0}, \quad x_{i}=X_{i} K_{1} / F_{0}, \quad i=1,2, \ldots, L,
\end{gathered}
$$

where $M_{i}$ is the mass, $K_{i}$ the stiffness, $C_{i}$ the damping, $F_{i}$ the force, $X_{i}$ the displacement of the $i$ th DOF, $\Omega$ the excitation frequency, $T$ at time, $B$ the clearance between $M_{1}$ and the point of impact, and $L$ the total number of DOF. The equation governing motion between impacts is

$$
\mathbf{M d}^{2} \mathbf{x} / \mathrm{d} t^{2}+\mathbf{C} \mathrm{d} \mathbf{x} / \mathrm{d} t+\mathbf{K} \mathbf{x}=\mathbf{f} \cos (\omega t+\theta),
$$

where $\mathbf{M}, \mathbf{C}, \mathbf{K}, \mathbf{f}, \mathbf{x}$ are matrices or vectors of the normalized quantities, and $\theta$ is the phase angle of the excitation. The conditions for impact are

$$
x_{1}\left(t_{1}\right)=b \quad \dot{x}_{1}\left(t_{i}^{-}\right)>0,
$$

where $t_{i}$ is the time at the $i$ th impact, and $t_{i}^{-}$is the moment just before impact, and the dot denotes differentiation with respect to time. The velocities before and after impact are related by the impact rule $\dot{x}_{1}\left(t_{i}^{+}\right)=-r \dot{x}_{1}\left(t_{i}^{-}\right)$, where $t_{i}^{+}$is the moment just after the $i$ th impact and $r$ is the coefficient of restitution and is an indication of the energy lost during impact. The impact is assumed to be instantaneous as far as the displacement is concerned, hence $x_{1}\left(t_{i}^{+}\right)=x_{1}\left(t_{i}^{-}\right)=x_{1}\left(t_{i}\right)=b$. The impact rule can be expressed as

$$
\left[\begin{array}{c}
\mathbf{x}\left(t_{i}^{+}\right) \\
\dot{\mathbf{x}}\left(t_{i}^{+}\right)
\end{array}\right]=\mathbf{L}\left[\begin{array}{c}
\mathbf{x}\left(t_{i}^{-}\right) \\
\dot{\mathbf{x}}\left(t_{i}^{-}\right)
\end{array}\right],
$$

where $\mathbf{L}$ is a diagonal matrix with elements equal to 1 , except that the element $1_{L+1}=-r$.

The goal here is to seek the periodic solutions of the impacting system. In general each response period may contain $N$ forcing periods and $P$ number of impacts. The first step of the solution process is to cast equation (2) into modal co-ordinates. It is assumed that proportional damping is used, so equation (2) can be reduced to

$$
\ddot{\mathbf{q}}+\boldsymbol{\Xi} \dot{\mathbf{q}}+\boldsymbol{\Lambda} \mathbf{q}=\mathbf{p} \cos (\omega t+\theta),
$$

where

$$
\begin{gathered}
\mathbf{q}=\left[q_{1}, q_{2}, \ldots, q_{L}\right]^{T}, \quad \mathbf{x}=\mathbf{U} \mathbf{q}, \\
\mathbf{\Lambda}=\operatorname{diag}\left[\omega_{i}^{2}\right], \quad \boldsymbol{\Xi}=\operatorname{diag}\left[2 \xi_{i} \omega_{i}\right], \quad \mathbf{p}=\mathbf{U}^{T} \mathbf{f}=\left[p_{1}, p_{2}, \ldots, p_{L}\right]^{T},
\end{gathered}
$$

$\mathbf{q}$ is a vector of modal co-ordinates and $\mathbf{U}$ is a matrix whose columns are the eigenvectors of the free vibration problem of equation (2), normalized with respect to the mass matrix. $\omega_{i}$ is the $i$ th eigenvalue, $\xi_{i}$ is the modal damping coefficient, and $\mathbf{p}$ is the modal load vector. The solution of equation (5) is

$$
\mathbf{q}=\mathbf{G a}+\mathbf{H b}+\mathbf{\Phi} \cos \theta+\boldsymbol{\psi} \sin \theta,
$$

where

$$
\begin{gathered}
\mathbf{G}=\operatorname{diag}\left[g_{i}\right], \quad \mathbf{H}=\operatorname{diag}\left[h_{i}\right], \\
\boldsymbol{\phi}=\left[\phi_{1}, \phi_{2}, \ldots, \phi_{L}\right]^{T}, \quad \boldsymbol{\psi}=\left[\psi_{1}, \psi_{2}, \ldots, \psi_{L}\right]^{T}, \\
\omega_{d i}=\omega_{i} \sqrt{1-\xi_{i}^{2}}, \\
g_{i}=\exp \left(-\xi_{i} \omega_{i} t\right) \sin \left(\omega_{d i} t\right), \quad h_{i}=\exp \left(-\xi_{i} \omega_{i} t\right) \cos \left(\omega_{d i} t\right),
\end{gathered}
$$




$$
\begin{aligned}
\phi_{i}=\alpha_{i} \cos \omega t+\beta_{i} \sin \omega t, & \psi_{i}=\beta_{i} \cos \omega t-\alpha_{i} \sin \omega t, \\
\alpha_{i}=\frac{\left(\omega_{i}^{2}-\omega^{2}\right) p_{i}}{\left(\omega_{i}^{2}-\omega^{2}\right)^{2}+2\left(\xi_{i} \omega_{i} \omega\right)^{2}}, & \beta_{i}=\frac{2 \xi_{i} \omega \omega_{i} \omega p_{i}}{\left(\omega_{i}^{2}-\omega^{2}\right)^{2}+2\left(\xi_{i} \omega \omega_{i} \omega\right)^{2}},
\end{aligned}
$$

and $\mathbf{a}, \mathbf{b}$ are yet to be determined constant vectors related to the initial conditions. The modal velocity vector can be expressed as

$$
\dot{\mathbf{q}}=\dot{\mathbf{G}} \mathbf{a}+\dot{\mathbf{H}} \mathbf{b}+\dot{\boldsymbol{\phi}} \cos \theta+\dot{\boldsymbol{\psi}} \sin \theta .
$$

Using equations (7), (11) and (23) to express $\mathbf{a}, \mathbf{b}$ in terms of the initial conditions, one finally has

$$
\left[\begin{array}{c}
\mathbf{x} \\
\dot{\mathbf{x}}
\end{array}\right]=\overline{\mathbf{U}} \mathbf{D} \overline{\mathbf{U}}^{-1}\left[\begin{array}{c}
\mathbf{x}_{0} \\
\dot{\mathbf{x}}_{0}
\end{array}\right]+\overline{\mathbf{U}} \boldsymbol{\Phi}\left[\begin{array}{c}
\cos \theta \\
\sin \theta
\end{array}\right],
$$

where

$$
\begin{gathered}
\overline{\mathbf{U}}=\left[\begin{array}{cc}
\mathbf{U} & \mathbf{0} \\
\mathbf{0} & \mathbf{U}
\end{array}\right], \quad \mathbf{D}=\left[\begin{array}{ll}
\mathbf{D}_{11} & \mathbf{D}_{12} \\
\dot{\mathbf{D}}_{11} & \mathbf{D}_{12}
\end{array}\right], \quad \boldsymbol{\Phi}=\left[\begin{array}{cc}
\overline{\boldsymbol{\Phi}} & \overline{\mathbf{\Psi}} \\
\overline{\overline{\boldsymbol{\Phi}}} & \dot{\mathbf{\Psi}}
\end{array}\right] \\
\overline{\boldsymbol{\Phi}=}\left[\bar{\phi}_{1}, \bar{\phi}_{2}, \ldots, \bar{\phi}_{L}\right]^{T}, \quad \overline{\mathbf{\Psi}}=\left[\bar{\psi}_{1}, \bar{\psi}_{2}, \ldots, \bar{\psi}_{L}\right]^{T}, \\
\bar{\phi}_{i}=\phi_{i}-\left(h_{i}+\xi_{i} g_{i} \omega_{i} / \omega_{d i}\right) \alpha_{i}-\beta_{i} g_{i} \omega / \omega_{d i}, \\
\bar{\psi}_{i}=\psi_{i}-\left(h_{i}+\xi_{i} g_{i} \omega_{i} / \omega_{d i}\right) \beta_{i}+\alpha_{i} g_{i} \omega / \omega_{d i}, \\
\mathbf{D}_{11}=\operatorname{diag}\left[h_{i}+\xi_{i} g_{i} \omega_{i} / \omega_{d i}\right], \quad \mathbf{D}_{12}=\operatorname{diag}\left[g_{i} / \omega_{d i}\right],
\end{gathered}
$$

where $\mathbf{x}_{0}, \dot{\mathbf{x}}_{0}$ are the initial displacement and velocity vectors, respectively. Defining

$$
\begin{gathered}
\mathbf{W}=\overline{\mathbf{U} D} \overline{\mathbf{U}}^{-1}, \quad \mathbf{V}=\overline{\mathbf{U} \Phi}, \\
\mathbf{y}=\left[x_{1}, \ldots, x_{L}, \dot{x}_{1}, \ldots, \dot{x}_{L}\right]^{T}=\left[\mathbf{x}^{T}, \dot{\mathbf{x}}^{T}\right]^{T}, \quad \mathbf{g}=[\cos \theta, \sin \theta]^{T},
\end{gathered}
$$

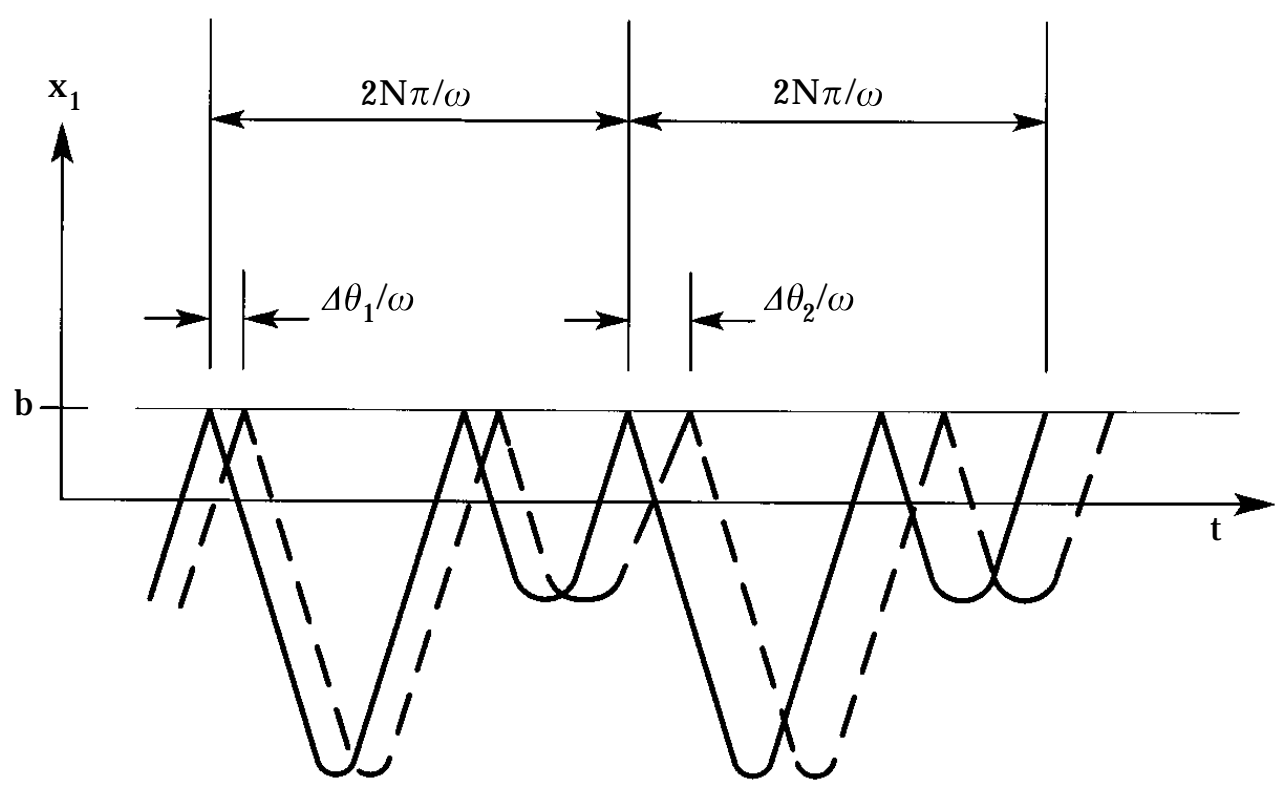

Figure 2. Local stability analysis. 

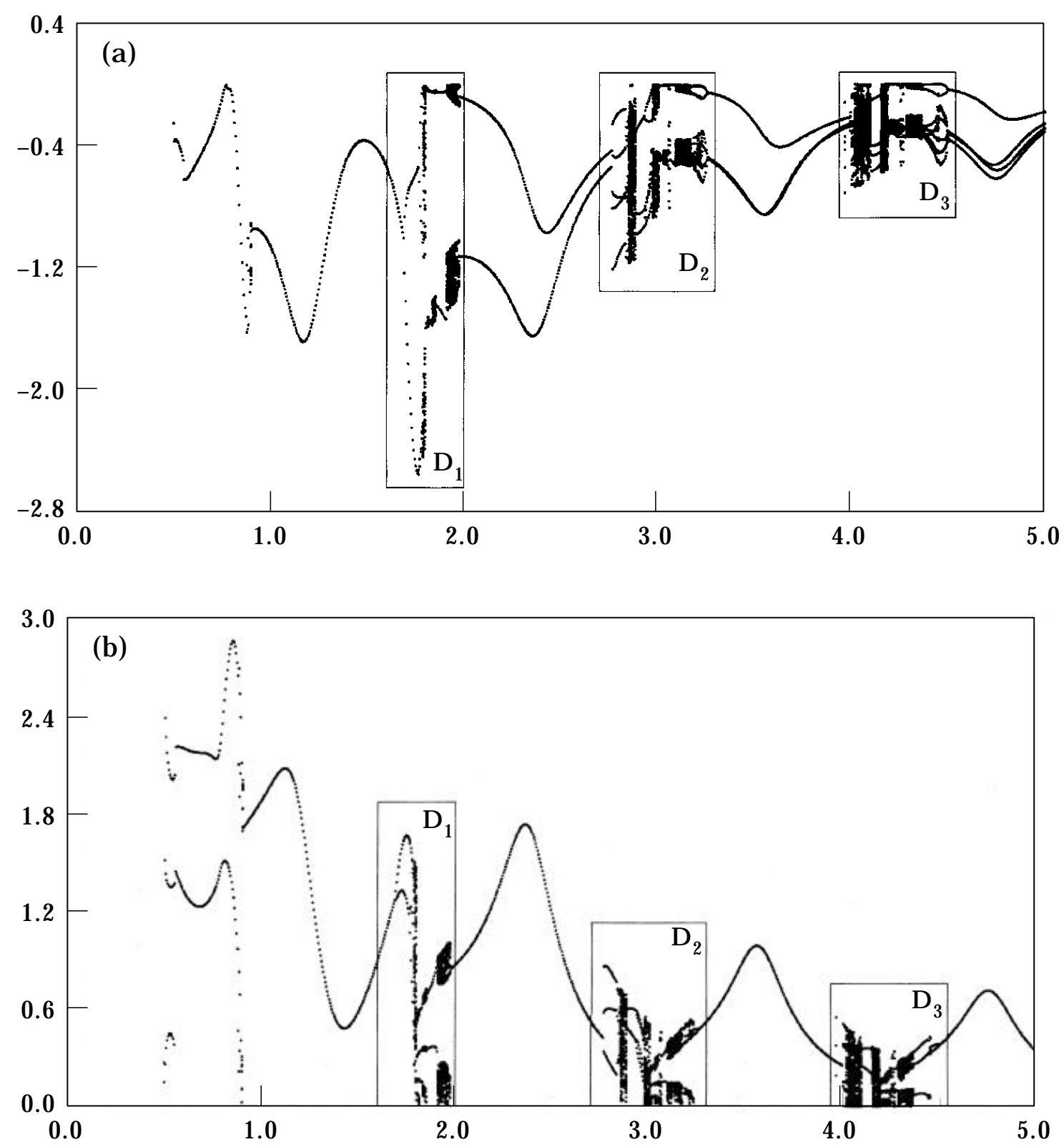

Figure 3. (a) Displacement $x_{1}$ versus frequency, $\omega<5$; (b) impact velocity $\dot{x}_{1}$ versus frequency, $\omega<5$.

then equation (24) can be recast with explicit time dependence, that is

$$
\mathbf{y}(t)=\mathbf{W}(t) \mathbf{y}(0)+\mathbf{V}(t) \mathbf{g}(\theta),
$$

where $\mathbf{y}(0)$ is a vector of initial displacements and velocities. To find periodic responses of $N$ forcing cycles and $P$ impacts, let the motion at the instant after an impact be used as a reference starting point. The time $t$ is set to zero and the initial conditions are $\mathbf{y}(0)$, $\theta$, where $y_{1}(0)=b$. Equation (38) governs the motion up to the next impact at time $t_{1}$, which can be solved by considering the equation for the first mass

$$
b=\sum_{n=1}^{2 L} W_{1 n}\left(t_{1}\right) y_{n}(0)+V_{11}\left(t_{1}\right) \cos \theta+V_{12}\left(t_{1}\right) \sin \theta .
$$

The motion after the first impact at $t_{1}$ is given by equations (4) and (38)

$$
\mathbf{y}^{1}(0)=\mathbf{L W}\left(t_{1}\right) \mathbf{y}(0)+\mathbf{L V}\left(t_{1}\right) \mathbf{g}(\theta),
$$




$$
\begin{gathered}
\theta_{1}=\omega t_{1}+\theta, \\
\mathbf{y}^{1}(t)=\mathbf{W}(t) \mathbf{y}^{1}(0)+\mathbf{V}(t) \mathbf{g}\left(\theta_{1}\right),
\end{gathered}
$$

where $\mathbf{y}^{1}(0)$ is the initial condition after the first impact, $\mathbf{y}^{1}(t)$ the subsequent motion up to the next impact, $t$ the time which begins from 0 after each impact, and $\theta_{1}$ the corresponding phase angle used to maintain force continuity. Similarly, the initial motion after $P$ impacts

$$
\mathbf{y}^{p}(0)=\mathbf{L W}\left(t_{p}\right) \mathbf{y}^{p-1}(0)+\mathbf{L V}\left(t_{p}\right) \mathbf{g}\left(\theta_{p-1}\right) .
$$

It is noted that equation (43) contains the impact times $t_{1}, t_{2}, \ldots, t_{p-1}$ implicitly through $\mathbf{y}^{p-1}(0)$. This relation can be written as

$$
\mathbf{y}^{p}(0)=\prod_{i=1}^{p}\left[\mathbf{L W}\left(t_{i}\right)\right] \mathbf{y}(0)+\sum_{j=1}^{p} \prod_{i=j+1}^{p}\left[\mathbf{L W}\left(t_{i}\right)\right]\left[\mathbf{L V}\left(t_{j}\right)\right] \mathbf{g}\left(\theta_{j-1}\right)
$$
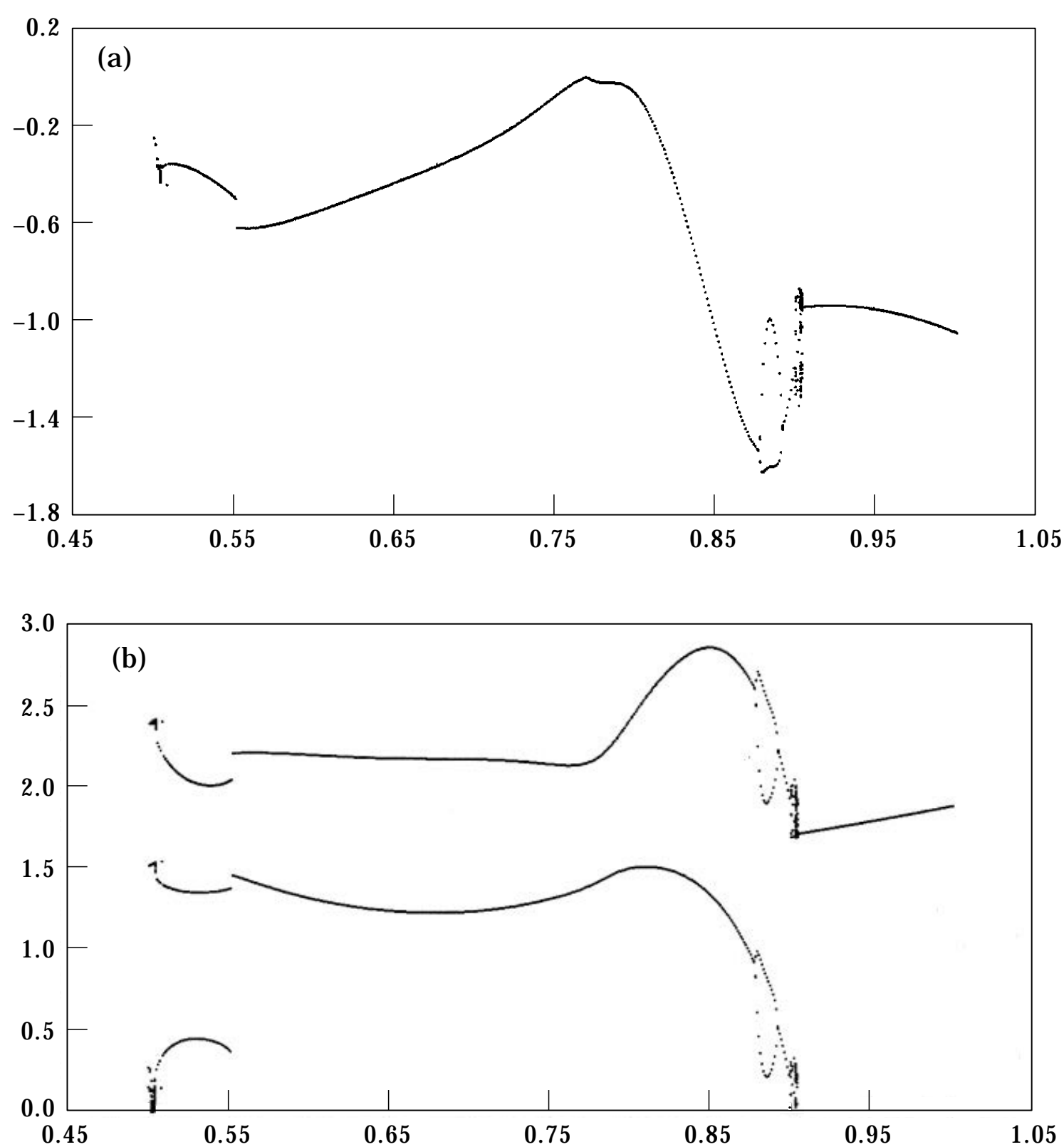

Figure 4. (a) Displacement $x_{1}$ versus frequency, $\omega<1 \cdot 05$. (b) impact velocity $\dot{x}_{1}$ versus frequency, $\omega<1.05$. 
where $\left[\mathbf{L W}\left(t_{i}\right)\right]$ and $\left[\mathbf{L V}\left(t_{j}\right)\right]$ are now treated as single matrices, and in the second term $\left[\mathbf{L W}\left(t_{i}\right)\right]=\mathbf{I}$ if $i>P$, and $\theta_{0}=\theta$. Using the periodic condition $\mathbf{y}^{p}(0)=\mathbf{y}(0)$, then $\mathbf{y}(0)$ can be solved in terms of $t_{i}(i=1, \ldots, P)$

$$
\mathbf{y}(0)=-\left[\prod_{i=1}^{p}\left[\mathbf{L W}\left(t_{i}\right)\right]-\mathbf{I}\right]^{-1}\left[\sum_{j=1}^{p} \prod_{i=j+1}^{p}\left[\mathbf{L W}\left(t_{i}\right)\right]\left[\mathbf{L V}\left(t_{j}\right)\right] \mathbf{g}\left(\theta_{j-1}\right)\right] .
$$
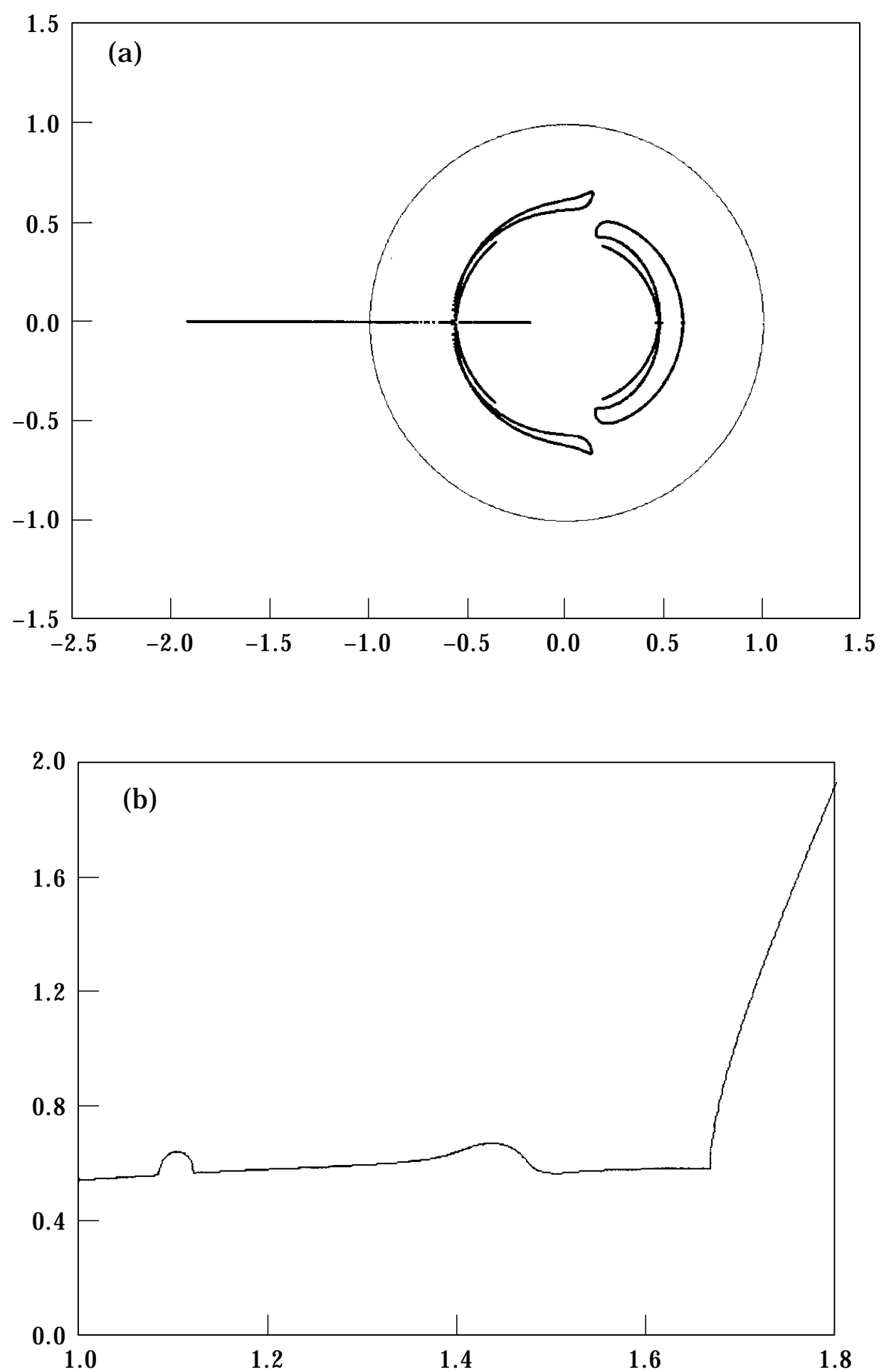

Figure 5. (a) Real and imaginary parts of eigenvalues, $1 \cdot 0 \leqslant \omega \leqslant 1 \cdot 8$; (b) spectral radius versus frequency. 

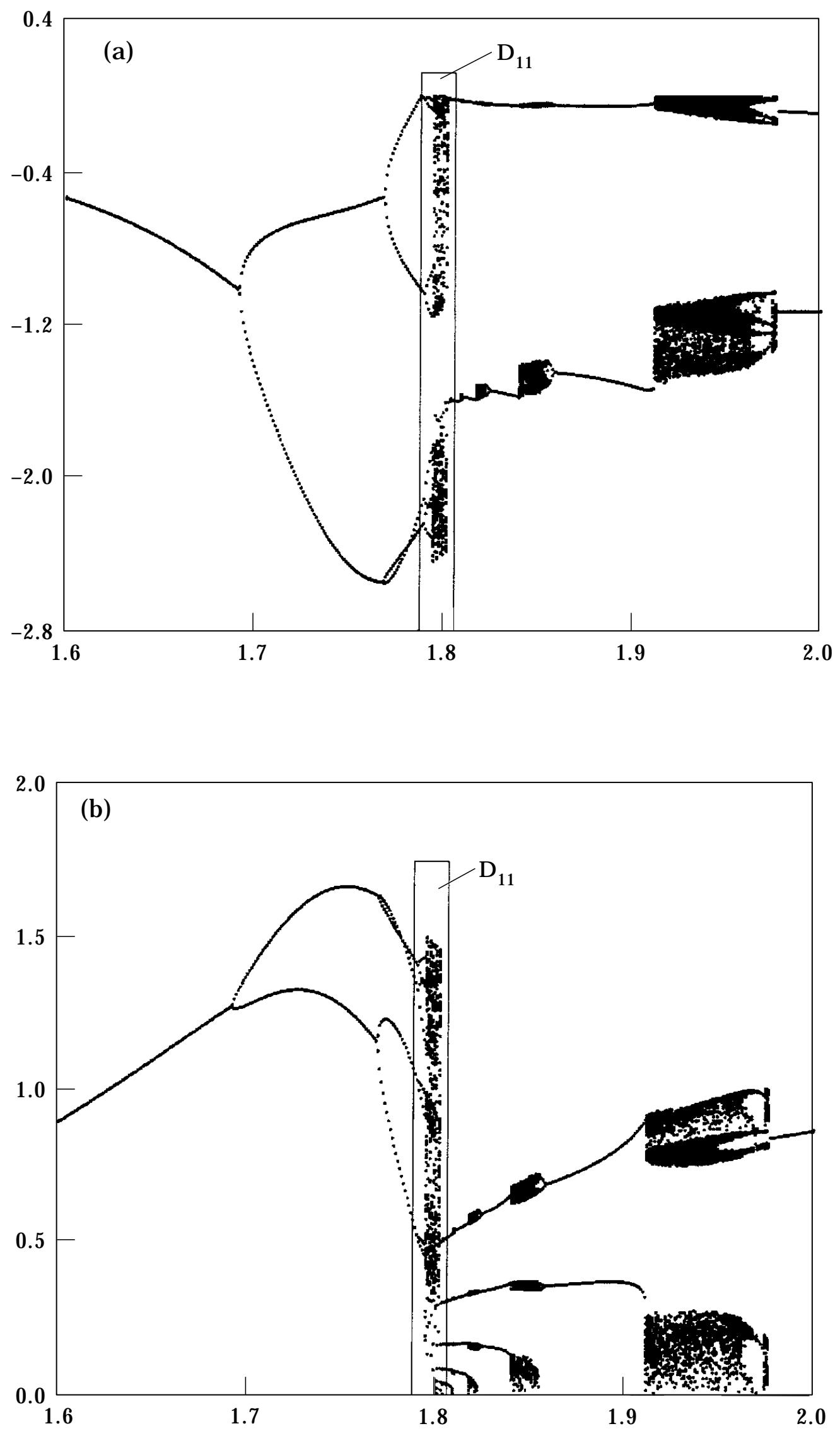

Figure 6. (a) Displacement $x_{1}$ versus frequency, $D_{1}$ window; (b) impact velocity $\dot{x}_{1}$ versus frequency, $D_{1}$ window. 
$\mathbf{y}(0)$ can then be substituted into the impact conditions, using the first row of the matrix equation (44), for the $k$ th impact

$$
\begin{gathered}
\mathbf{y}_{1}^{k}(0)=b=\sum_{m=1}^{2 L}\left(\prod_{i=1}^{k}\left[\mathbf{L W}\left(t_{i}\right)\right]\right)_{1 m} y_{m}(0)+\sum_{m=1}^{2 L}\left(\sum_{j=1}^{k} \prod_{i=j+1}^{k}\left[\mathbf{L W}\left(t_{i}\right)\right]\left[\mathbf{L V}\left(t_{j}\right)\right]\right)_{1 m} g_{m}\left(\theta_{j-1}\right), \\
k=1,2, \ldots, P
\end{gathered}
$$

where subscripted brackets indicate matrix element of the enclosed quantity. As there are $P$ impacts, $P$ equations can be set up on this basis. The variables $\theta_{i}(i=1, \ldots, P-1)$ can be expressed in terms of $t_{i}(i=1, \ldots, P-1)$ by the relation

$$
\theta_{k}=\omega t_{k}+\theta_{k-1}, \quad k=1,2, \ldots, P,
$$

and note that $t_{p}$ can be written as

$$
t_{p}=\frac{2 N \pi}{\omega}-\sum_{i}^{p-1} t_{i}
$$

so that the final unknowns are $t_{i}(i=1, \ldots, P-1)$ and $\theta$. With the initial conditions given, equation (46) furnishes a set of simultaneous non-linear equations that can be used to solve for $N-P$ motion. The process is doubly iterative, for in addition to being non-linear in the unknown variables, the values of $N$ and $P$ are not known beforehand and have to be assumed.

\subsection{STABILITY ANALYSIS}

The local stability analysis is carried out as illustrated in Figure 2, where the original initial conditions are perturbed to an adjacent state $\tilde{\mathbf{y}}(0)$, given by

$$
\tilde{\mathbf{y}}(0)=\mathbf{y}(0)+\Delta \mathbf{y}(0) \text {. }
$$
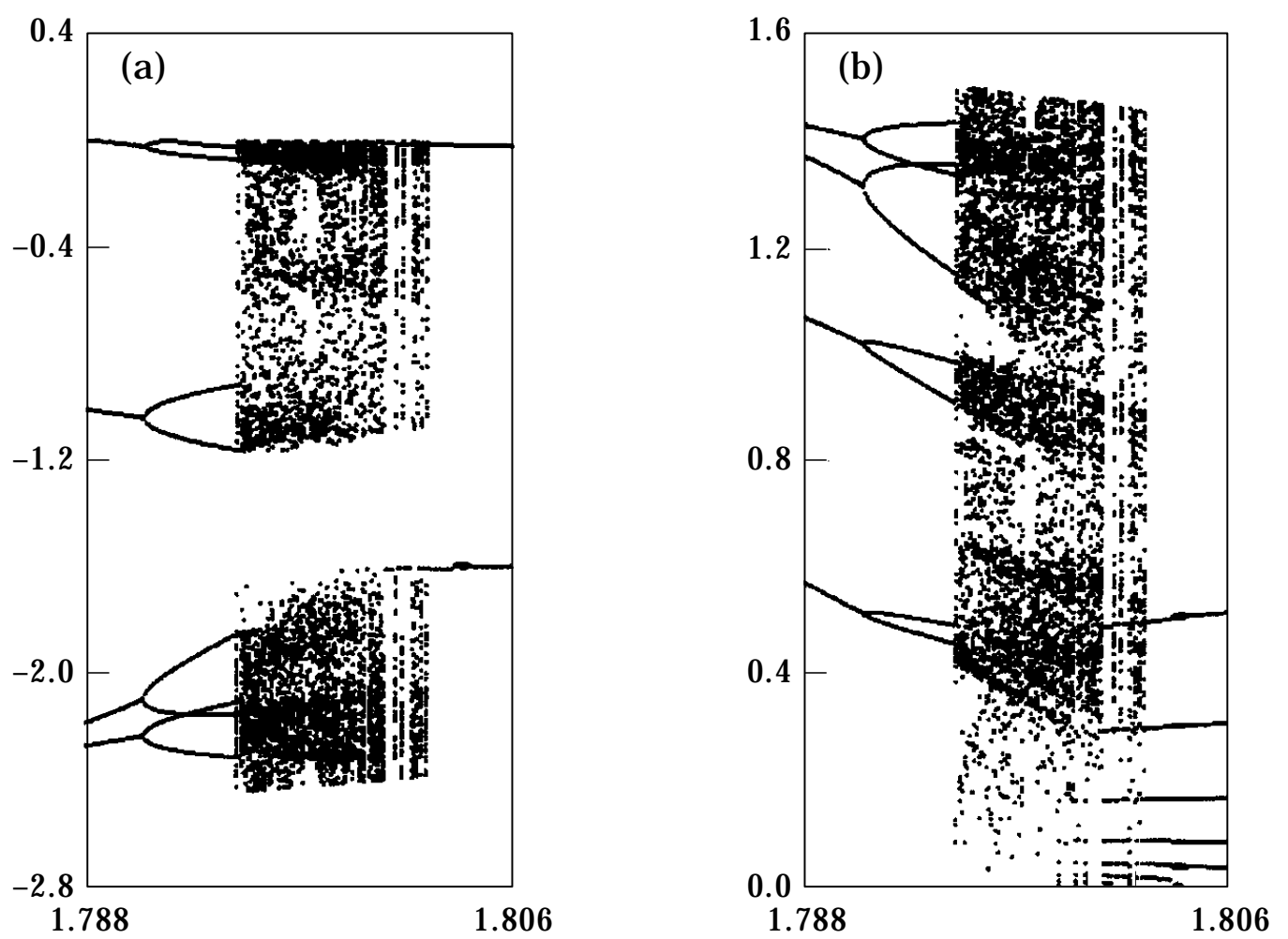

Figure 7. (a) Displacement $x_{1}$ versus frequency, $D_{11}$ window; (b) impact velocity $\dot{x}_{1}$ versus frequency, $D_{11}$ window. 

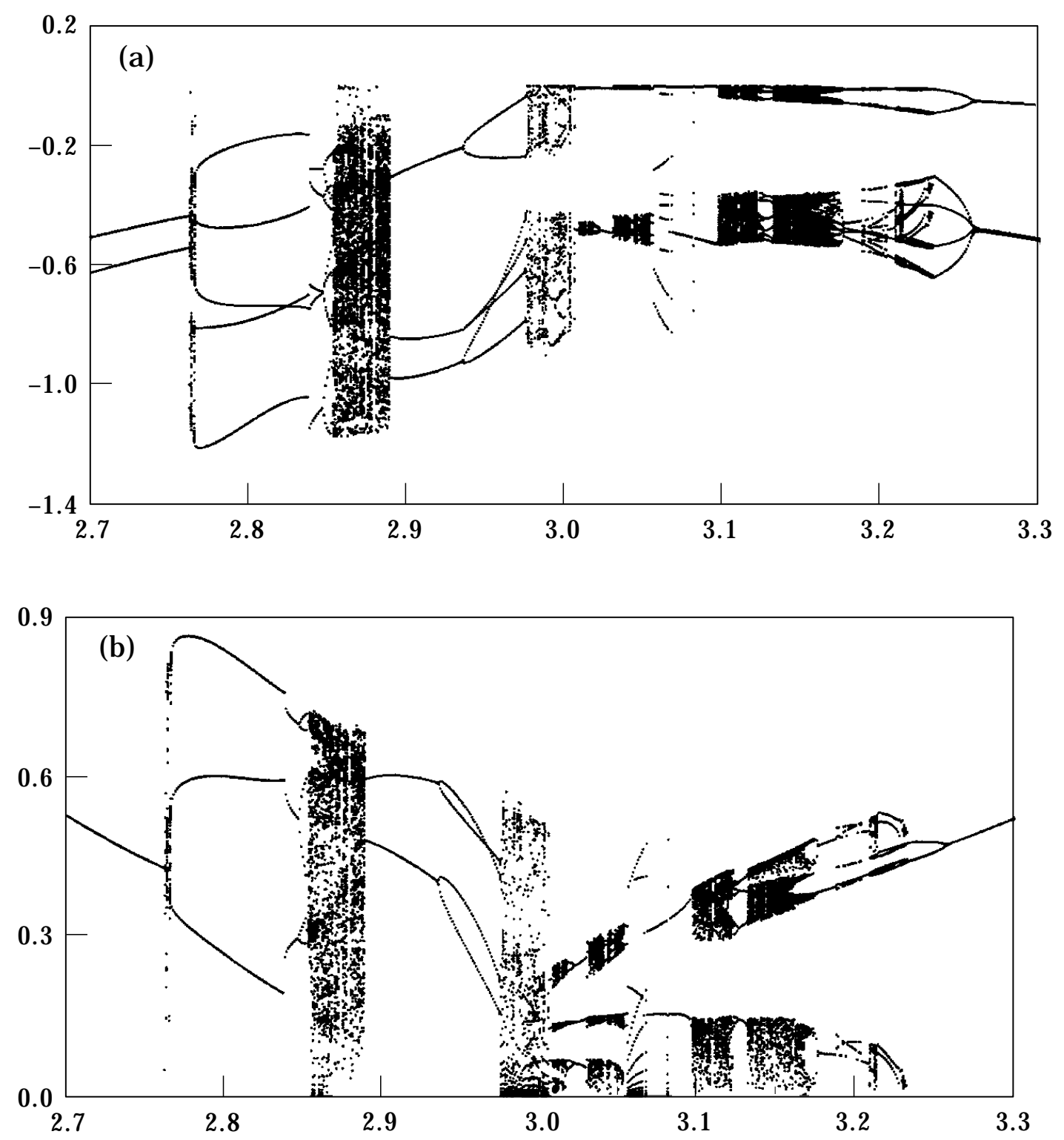

Figure 8. (a) Displacement $x_{1}$ versus frequency, $D_{2}$ window; (b) impact velocity $\dot{x}_{1}$ versus frequency, $D_{2}$ window.

From equation (36), it follows that

$$
\Delta \mathbf{y}(0)=[\Delta \mathbf{x}(0) \Delta \dot{\mathbf{x}}(0)]^{T} .
$$

As the reference state is at the moment just after an impact, it is clear that $x_{1}(0)=b$ and $\Delta x_{1}(0)=0$. In place of $\Delta x_{1}(0)$, the impact time is subject to variation and this is incorporated by varying the phase angle $\theta$. Taking variation of equation (38) gives

$$
\Delta \mathbf{y}(t)=\mathbf{W}(t) \Delta \mathbf{y}(0)+\mathbf{V}(t) \mathbf{g}^{\prime}(\theta) \Delta \theta+[\dot{\mathbf{W}}(t) \mathbf{y}(0)+\dot{\mathbf{V}}(t) \mathbf{g}(\theta)] \Delta t,
$$

where

$$
\mathbf{g}^{\prime}(\theta)=[-\sin \theta, \cos \theta]^{T} .
$$

The initial deviations after the first impact are obtained by applying equation (4) to equation (51)

$$
\Delta \mathbf{y}^{1}(0)=\left[\mathbf{L W}\left(t_{1}\right)\right] \Delta \mathbf{y}(0)+\left[\mathbf{L V}\left(t_{1}\right)\right] \mathbf{g}^{\prime}(\theta) \Delta \theta+\mathbf{L}\left[\dot{\mathbf{W}}\left(t_{1}\right) \mathbf{y}(0)+\dot{\mathbf{V}}\left(t_{1}\right) \mathbf{g}(\theta)\right] \Delta t_{1} .
$$


It can be appreciated that $\left[\mathbf{L V}\left(t_{1}\right)\right] \mathbf{g}^{\prime}(\theta)$ and $\mathbf{L}\left[\dot{\mathbf{W}}\left(t_{1}\right) \mathbf{y}(0)+\dot{\mathbf{V}}\left(t_{1}\right) \mathbf{g}(\theta)\right]$ are column vectors. The first row of this matrix equation is

$$
\Delta y_{1}^{1}(0)=\sum_{i=1}^{2 L}\left[\mathbf{L W}\left(t_{1}\right)\right]_{1 i} \Delta y_{i}(0)+\left[\mathbf{L} \mathbf{V}\left(t_{1}\right) \mathbf{g}^{\prime}(\theta)\right]_{1} \Delta \theta+\left\{\mathbf{L}\left[\dot{\mathbf{W}}\left(t_{1}\right) \mathbf{y}(0)+\dot{\mathbf{V}}\left(t_{1}\right) \mathbf{g}(\theta)\right]\right\}_{1} \Delta t_{1},
$$

noting that $\Delta y_{1}^{1}(0)=\Delta y_{1}(0)=0, \Delta t_{1}$ can be found

$$
\Delta t_{1}=\frac{-\sum_{i=2}^{2 L}\left[\mathbf{L W}\left(t_{1}\right)\right]_{1 i} \Delta y_{i}(0)+\left[\mathbf{L V}\left(t_{1}\right) \mathbf{g}^{\prime}(\theta)\right]_{1} \Delta \theta}{\left\{\mathbf{L}\left[\dot{\mathbf{W}}\left(t_{1}\right) \mathbf{y}(0)+\dot{\mathbf{V}}\left(t_{1}\right) \mathbf{g}(\theta)\right]\right\}_{1}} .
$$

The relationship between the time and phase variations can be inferred from equation (47)

$$
\Delta \theta_{1}=\omega \Delta t_{1}+\Delta \theta .
$$
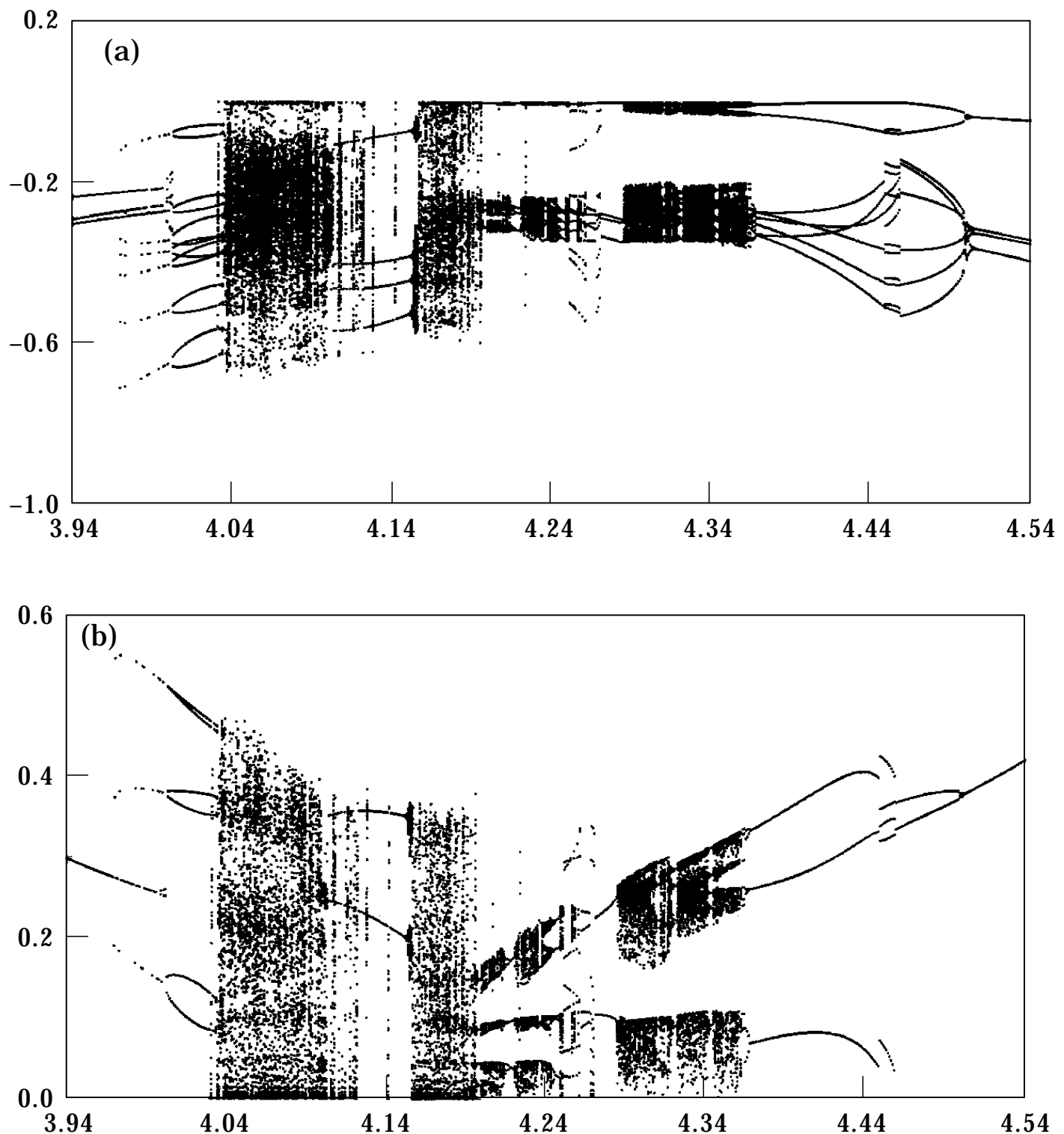

Figure 9. (a) Displacement $x_{1}$ versus frequency, $D_{3}$ window; (b) impact velocity $\dot{x}_{1}$ versus frequency, $D_{3}$ window. 


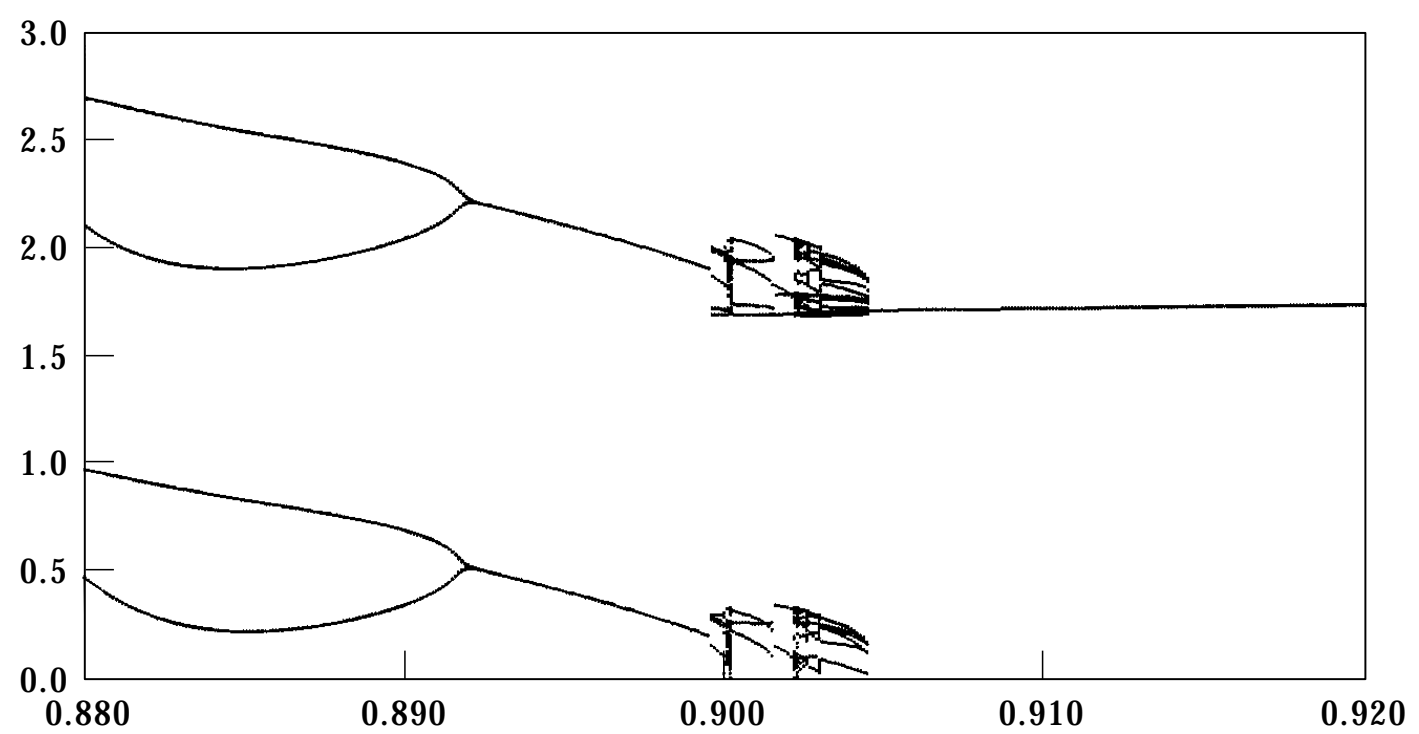

Figure 10 . Impact velocity $\dot{x}_{1}$ versus frequency, $\omega \approx 0.9$.

Equation (55) can be substituted into equation (56) to obtain a relation for $\Delta \theta_{1}$ in terms of $\Delta y_{i}(0)(i=2, \ldots, 2 L)$ and $\Delta \theta$. It should also be apparent that by using equations (55) and (56) and the 2 to $2 L$ rows of equation (53), one can obtain the expression

$$
\left[\begin{array}{c}
\Delta y_{2}^{1} \\
\cdot \\
\cdot \\
\cdot \\
\cdot \\
\Delta y_{2 L}^{1} \\
\Delta \theta_{1}
\end{array}\right]=\mathbf{G}_{0}\left[\begin{array}{c}
\Delta y_{2} \\
\cdot \\
\cdot \\
\cdot \\
\cdot \\
\Delta y_{2 L} \\
\Delta \theta
\end{array}\right]=\mathbf{G}_{0} \mathbf{z},
$$

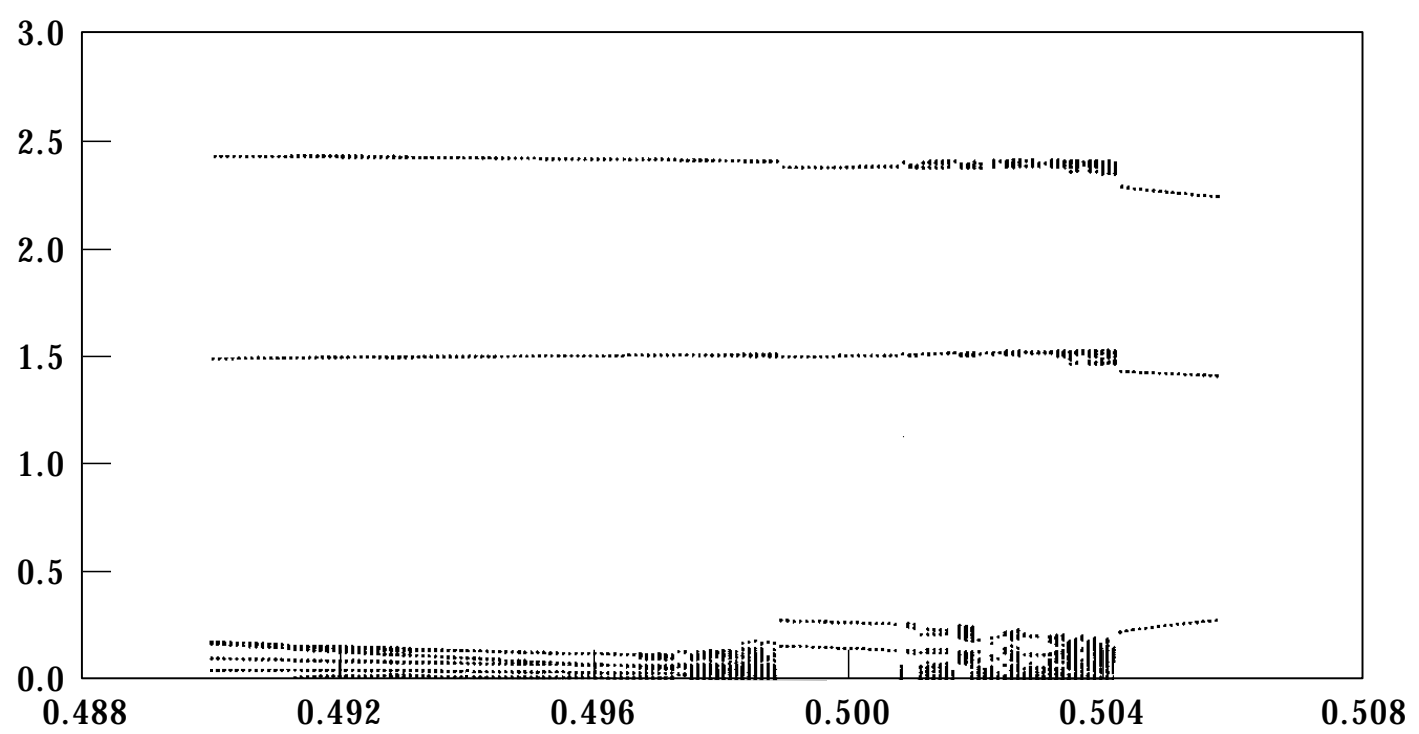

Figure 11. Impact velocity $\dot{x}_{1}$ versus frequency, $\omega \approx 0 \cdot 5$. 
(a)
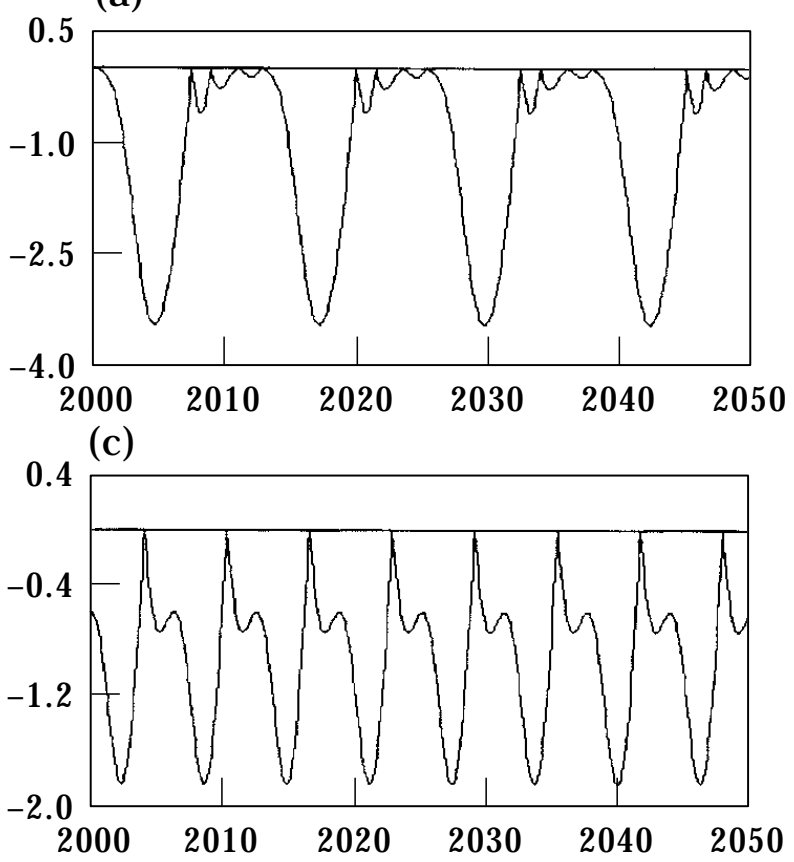

(e) (b)

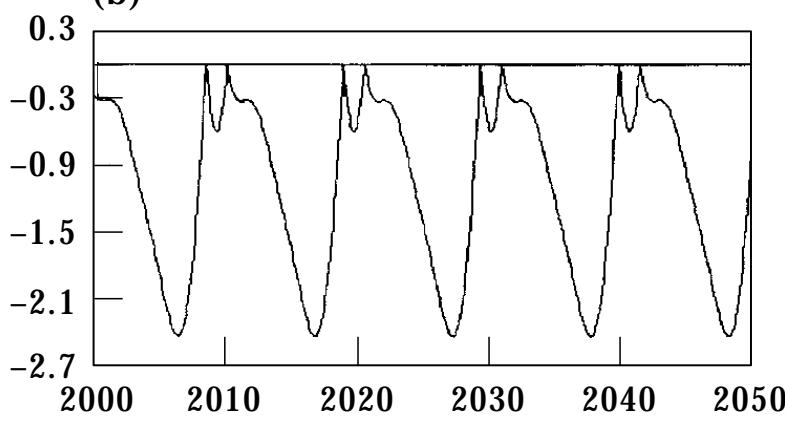

(d)

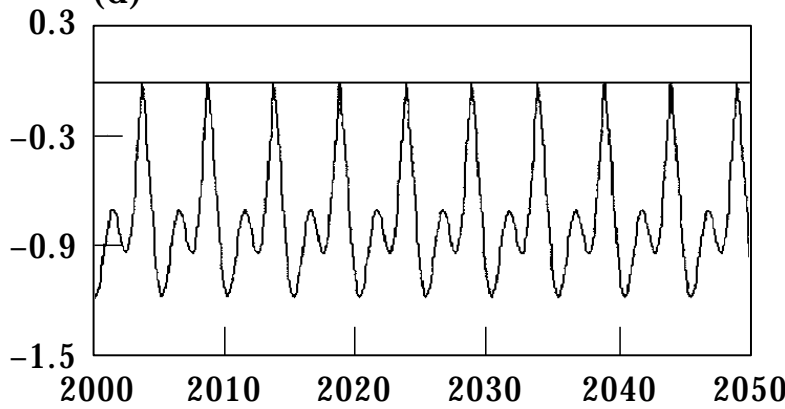

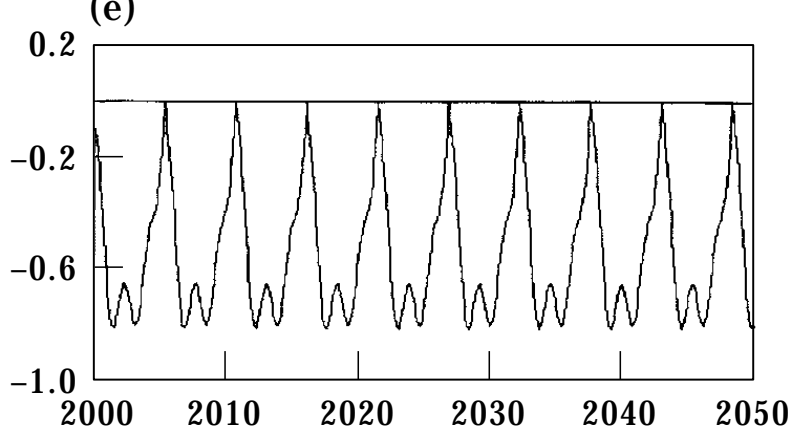

Figure 12. Displacement $x_{1}$ versus time: (a) 1-4 motion, $\omega=0 \cdot 50$; (b) 1-2 motion, $\omega=0 \cdot 60$; (c) $1-1$ motion, $\omega=1 \cdot 00$; (d) 2-1 motion, $\omega=2 \cdot 50$; (c) 3-1 motion, $\omega=3 \cdot 50$.

where the matrix $\mathbf{G}_{0}$ gives the variations after the first impact in terms of the initial ones. This procedure can be carried out $P$ times to arrive at the motion deviations after $P$ impact occurrences. Defining

$$
\mathbf{G}=\mathbf{G}_{P-1} \cdots \mathbf{G}_{1} \mathbf{G}_{0}
$$

then

$$
\mathbf{z}_{1}=\mathbf{G z},
$$

where $\mathbf{z}_{1}$ is the variation at the end of the one response cycle and $\mathbf{G}$ is the transition matrix. The variations after $k$ cycles are

$$
\mathbf{z}_{k}=\mathbf{G}^{k} \mathbf{z},
$$

and the stability of a periodic solution is determined by the eigenvalues of the transition matrix. The case of interest in this study is the flip or period doubling bifurcation, where an eigenvalue becomes -1 and leaves the unit circle along the real axis.

\section{RESULTS}

A system with the following properties is analysed below:

$$
\mathbf{M}=\left[\begin{array}{ll}
1 & 0 \\
0 & 1
\end{array}\right], \quad \mathbf{K}=\left[\begin{array}{cc}
1 & -1 \\
-1 & 2
\end{array}\right], \quad \mathbf{C}=\left[\begin{array}{cc}
0 \cdot 05 & -0 \cdot 05 \\
-0 \cdot 05 & 0 \cdot 1
\end{array}\right], \quad \mathbf{f}=\left[\begin{array}{l}
1 \\
0
\end{array}\right] .
$$


The coefficient of restitution is $0 \cdot 6$, the gap $b$ is 0 , and the system natural frequencies are $\omega_{1}=0.618$ and $\omega_{2}=1.618$. The initial conditions are

$$
\mathbf{x}=\left[\begin{array}{l}
-1 \\
-1
\end{array}\right], \quad \dot{\mathbf{x}}=\left[\begin{array}{l}
1 \\
1
\end{array}\right]
$$

The solutions are obtained from equations (38), (39) and (43); the first 1800 normalized secs of the responses are ignored, and the motion of the next 100 impacts are recorded. The initial conditions are fixed at the specified values as the solution branches are traced out over the frequency spectrum. Figures 3(a) and (b) show the displacement and impact velocity spectra for the frequency range $0-5$. As the system's state space is five-dimensional, the Poincare sections for the displacement and impact velocity spectra are four-dimensional. The displacement section is taken at once per period of the excitation whereas the impact velocity is sampled when $x_{1}=0$ and $\dot{x}_{1}>0$, where impact occurs. Hence, the number of branches in the displacement spectrum corresponds to $N$ forcing cycles, whereas those in the velocity spectrum give $P$ impacts. It can be seen from Figure 3 that relatively narrow windows of chaotic motion are imbedded between regimes of harmonic response.

The chaotic windows, labelled $D$, are near the integer values of frequency, but it is possible to discern that the windows are gradually displaced to higher frequency slots as

(a)
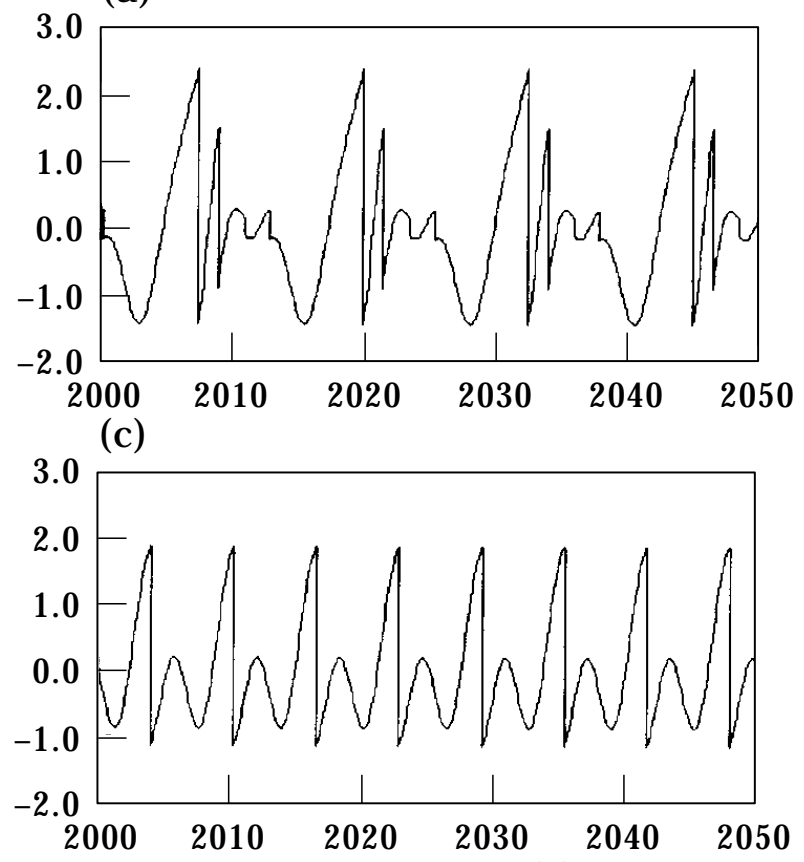

(b)
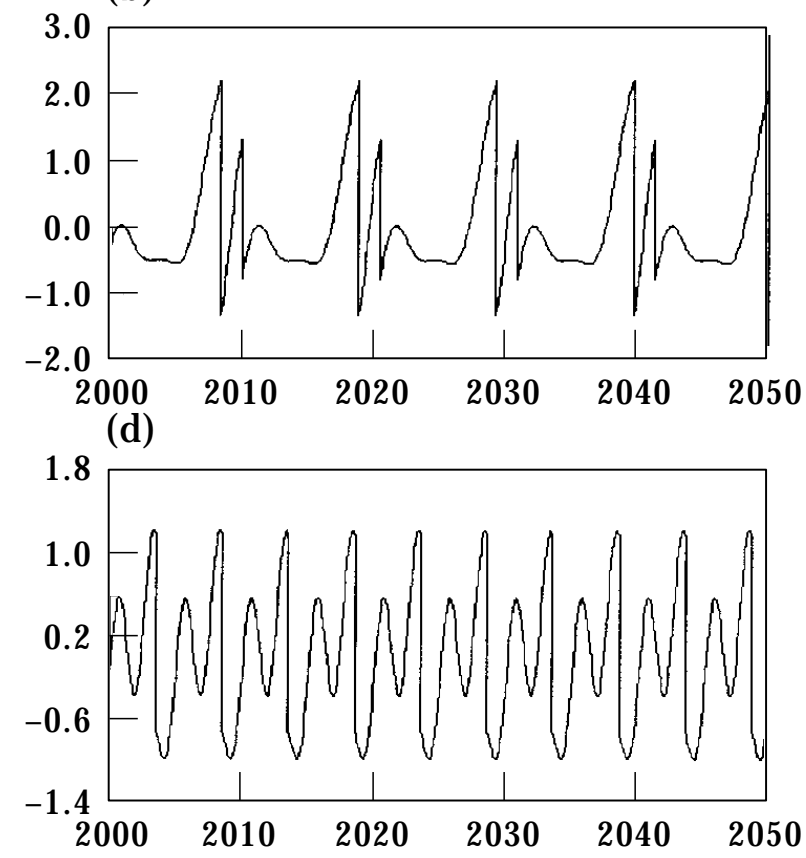

(e)

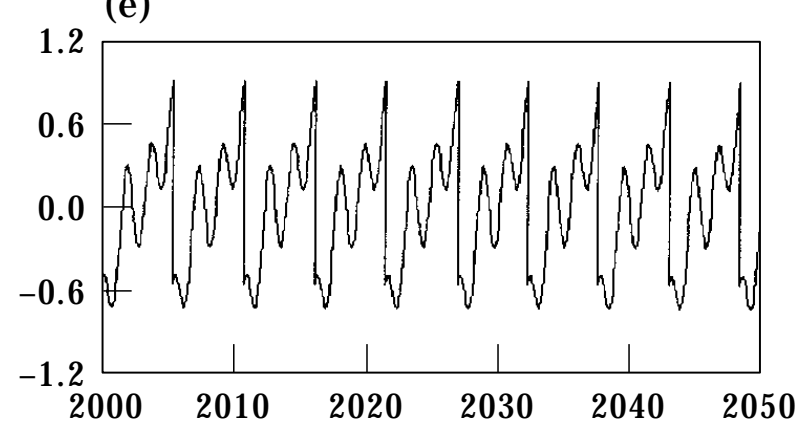

Figure 13. Velocity $\dot{x}_{1}$ versus time: (a) 1-4 motion, $\omega=0 \cdot 50$; (b) $1-2$ motion, $\omega=0 \cdot 60$; (c) 1-1 motion, $\omega=1 \cdot 00$; (d) $2-1$ motion, $\omega=2 \cdot 50$; (c) $3-1$ motion, $\omega=3 \cdot 50$. 
(a)

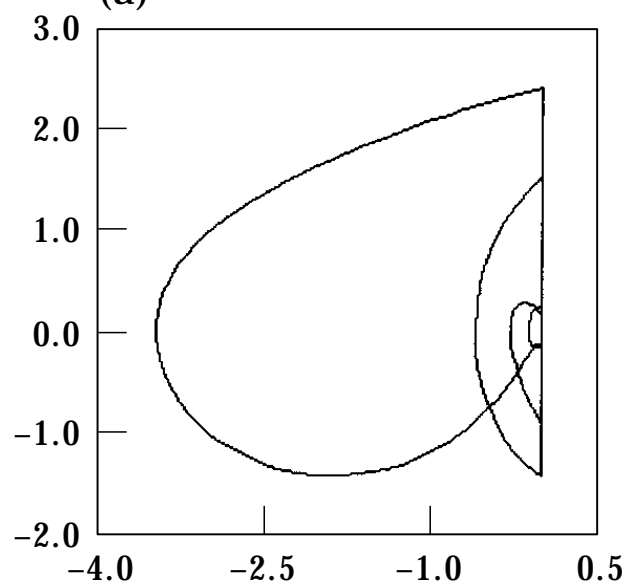

(c)

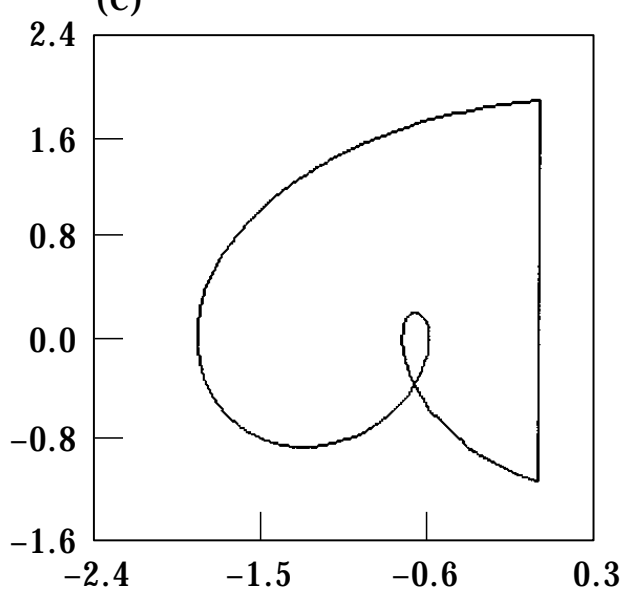

(b)

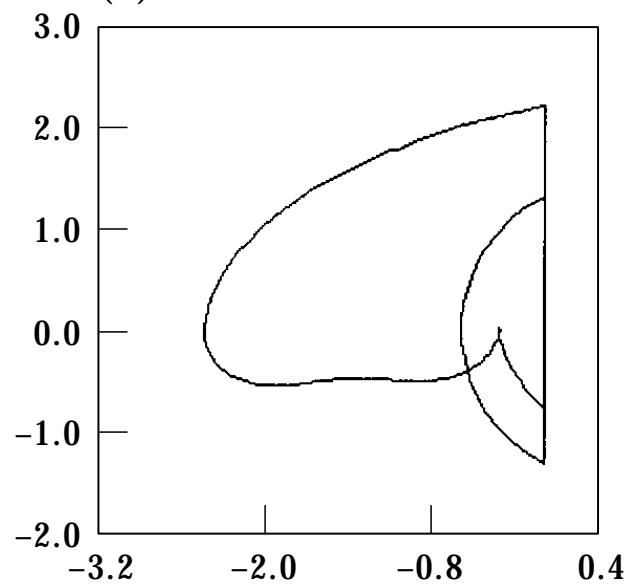

(d)

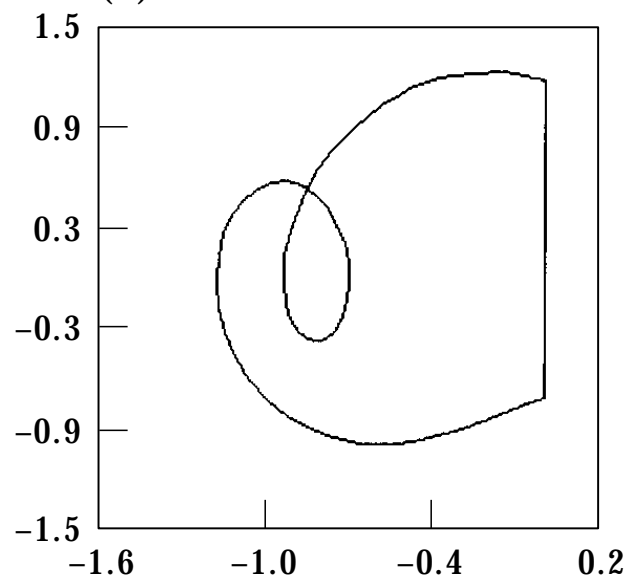

(e)

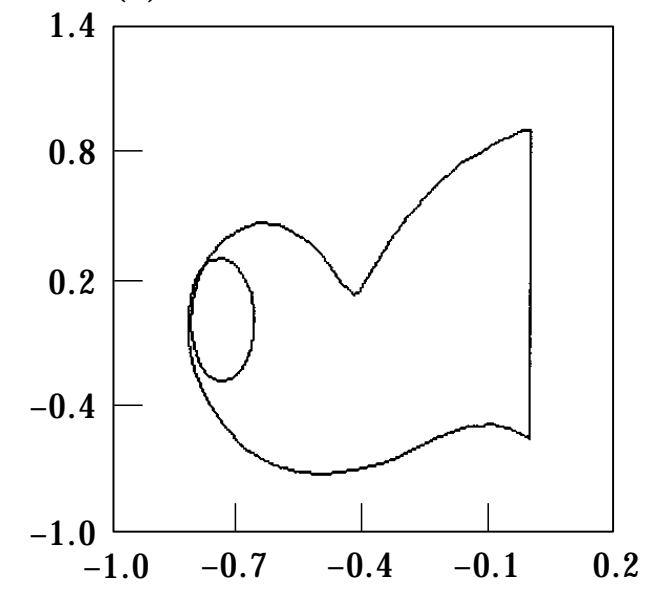

Figure 14. Phase diagrams, $\dot{x}_{1}$ versus $x_{1}$ : (a) 1-4 motion, $\omega=0 \cdot 50$; (b) $1-2$ motion, $\omega=0 \cdot 60$; (c) 1-1 motion, $\omega=1 \cdot 00$; (d) 2-1 motion, $\omega=2 \cdot 50$; (c) 3-1 motion, $\omega=3 \cdot 50$.

the frequency is increased. Furthermore, the width of the chaotic windows seems to increase with the frequency. Outside the chaotic windows, $N-1$ motions, where $N$ seems to equal the adjacent lower integer frequency, are prevalent and these become chaotic by undergoing a series of period-doubling bifurcations.

Figures 4(a) and (b) show the displacement and impact velocity spectra for frequencies of 0.45 to 1 . Here, again, chaotic slots are embedded into broader regions of periodic response. The prevailing periodic responses are of the 1-P type, where $P$ ranges from 1 to 3 and increases with decreasing frequency. The response here is qualitatively the same as that in Figure 3, but the results show that chaotic regions are not necessarily close to integer values of frequency. 
There is period one motion at the natural frequencies of 0.618 and $1 \cdot 618$, as might be intuitively expected. As the frequency increases, the impact velocity decreases. For excitation frequency larger than one, motions with $N$ generally larger than $P$ are present in the periodic regimes, and the reverse is true for the freuqency region less than one.

The first bifurcation takes place at a frequency of $1 \cdot 69$, according to the time integration results. This confirmed by the stability analysis, which shows that an eigenvalue of the transition matrix $\mathbf{G}$ leaves the unit circle at -1.0 within the frequency range of 1 to $1 \cdot 8$, as shown in Figure 5(a). Furthermore, a plot of the spectral radius in Figure 5(b) indicates that the bifurcation is at the frequency of 1.69 .

The window $D_{1}$ as shown in Figures 6(a) and (b) contains more details on the bifurcation behaviour. The 2-1 motion undergoes a sequence of period-doubling bifurcations, with the longest discernable motion being 8-8, on the road to chaos in window $D_{11}$. The chaotic band $D_{11}$ at frequency $1 \cdot 8$, as shown in Figure 7 , is characterized by high displacement and

(a)

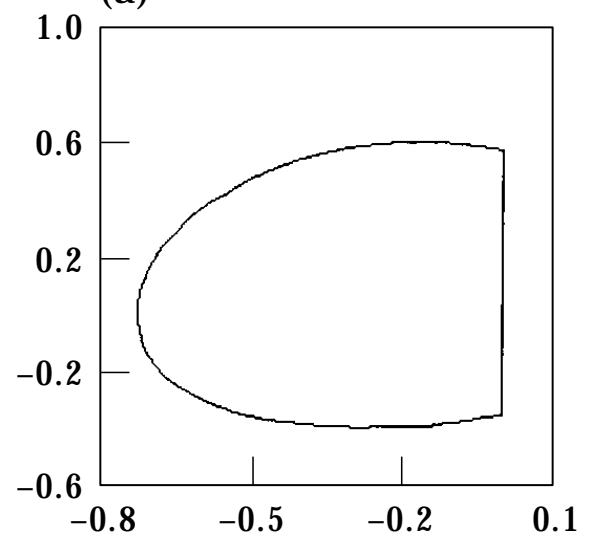

(c)

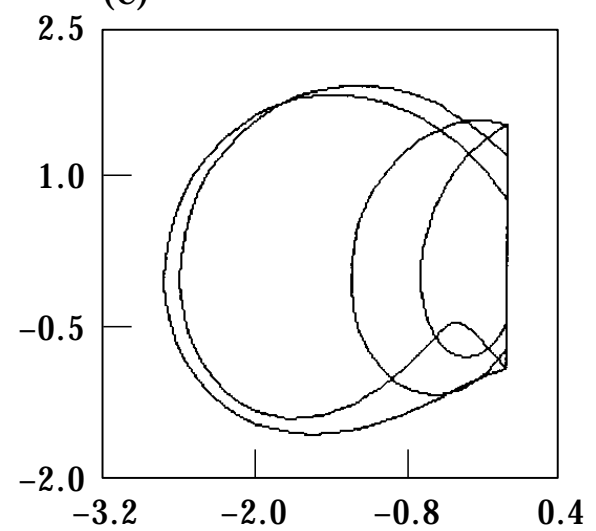

(e)

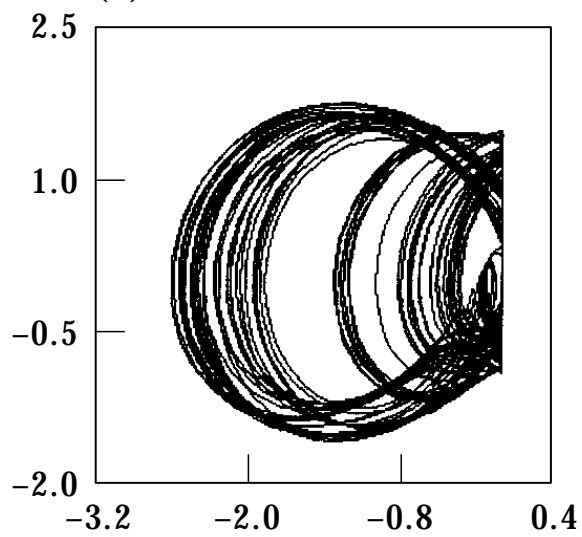

(b)

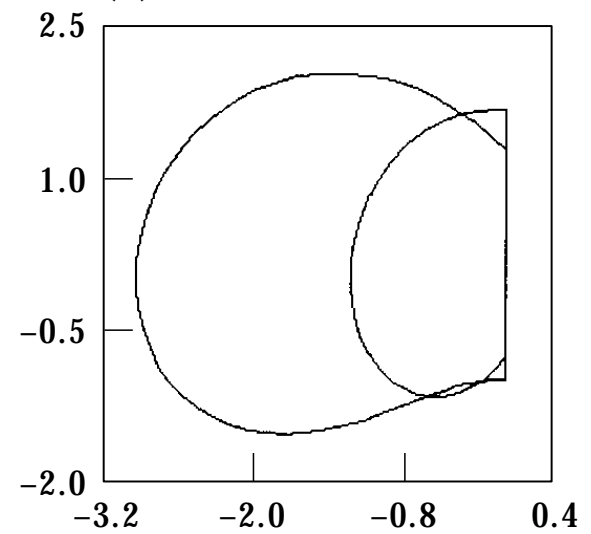

(d)

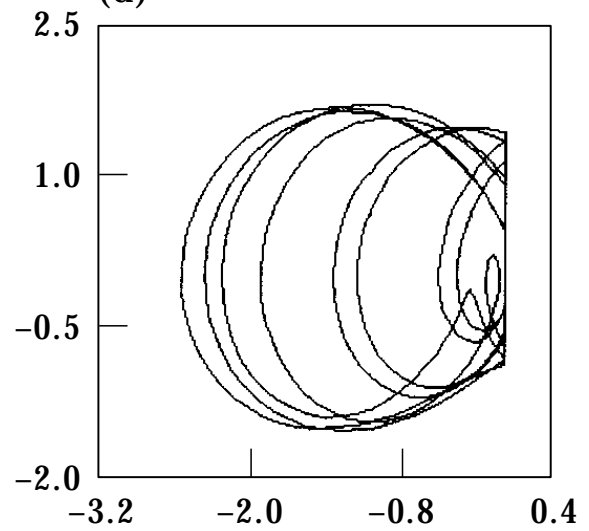

(f)

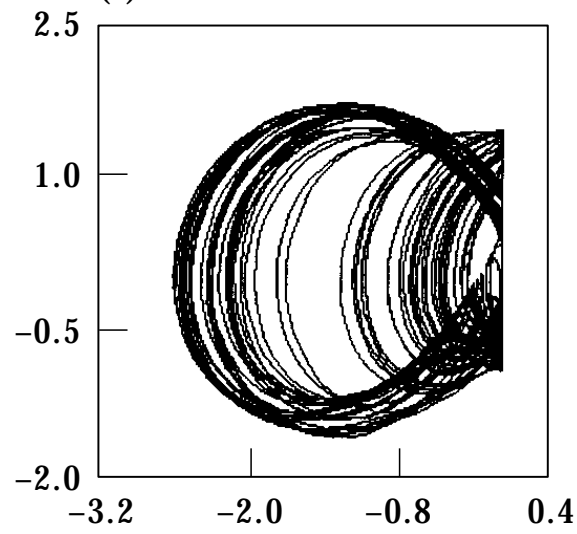

Figure 15. Phase diagrams showing route to chaos, $\dot{x}_{1}$ versus $x_{1}$ : (a) $\omega=1 \cdot 500$; (b) $\omega=1 \cdot 750 ;(c) \omega=1 \cdot 780$; (d) $\omega=1.794$; (e) $\omega=1.796$; (f) $\omega=1.800$. 
(a)

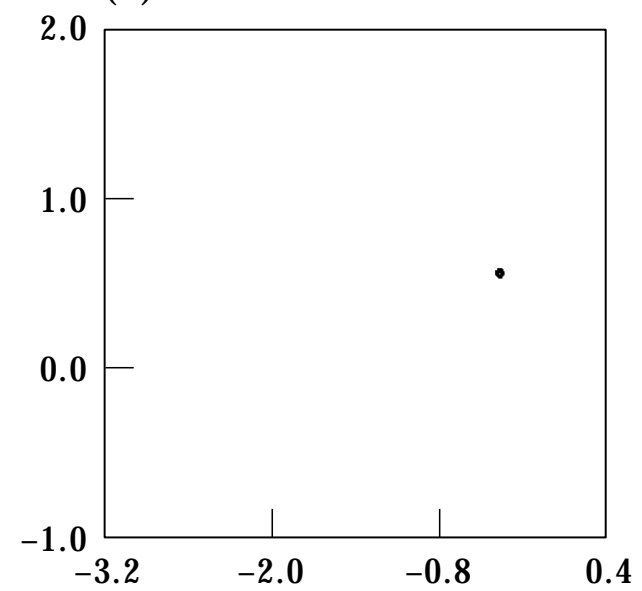

(c)

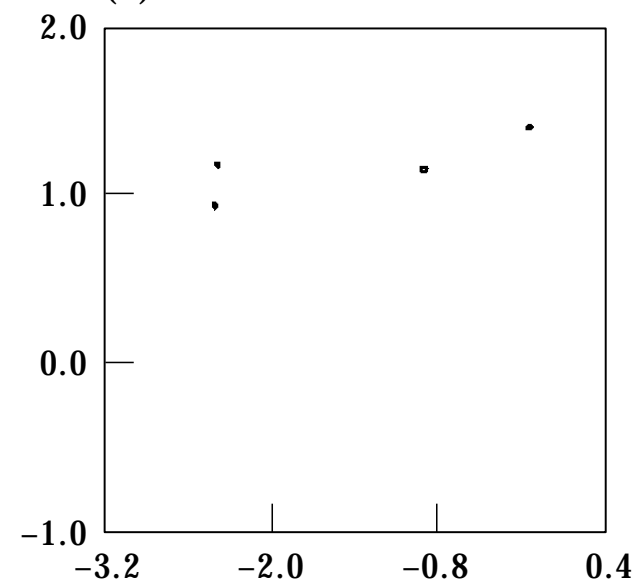

(e)

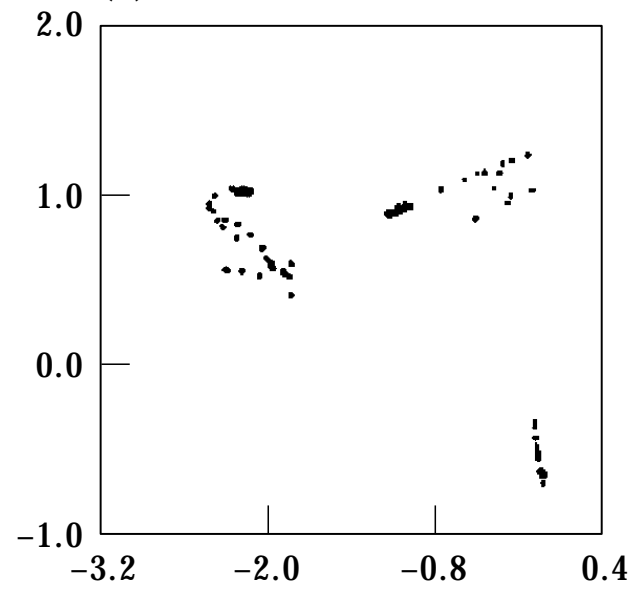

(b)

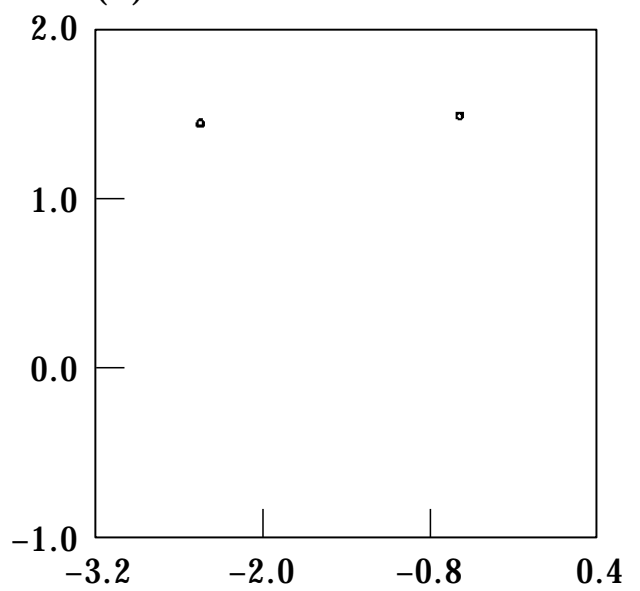

(d)

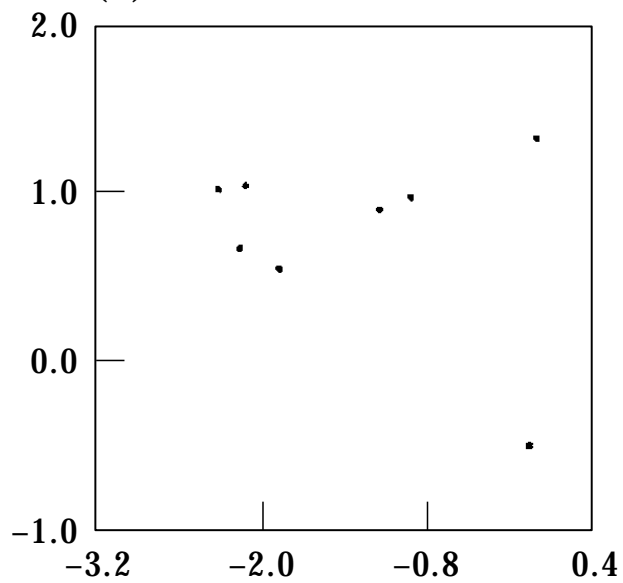

(f)

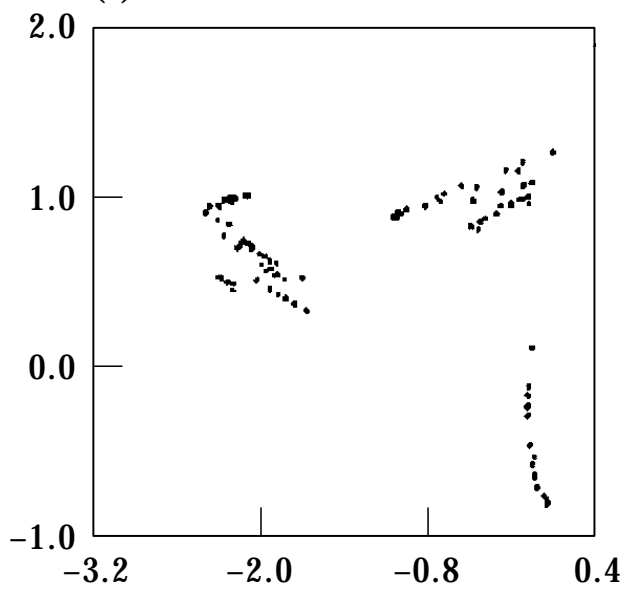

Figure 16. Poincaré maps showing route to chaos, $\dot{x}_{1}$ versus $x_{1}$ : (a) $\omega=1 \cdot 500$; (b) $\omega=1 \cdot 750$; (c) $\omega=1 \cdot 780$; (d) $\omega=1 \cdot 794$; (e) $\omega=1 \cdot 796$; (f) $\omega=1.800$.

velocity excursions. Two more chaotic windows, as shown in Figures 8 and 9, exist at higher frequencies. Both the displacement and velocity excursions show decreasing magnitudes with increasing frequency, just as in the case for the periodic regimes. The chaotic windows $D_{2}$ and $D_{3}$ at higher frequencies have progressively higher bandwidths, contain more periodic-doubling bifurcations, and more chaotic bands. Low velocity or grazing impacts, indicative of movement close to the wall, are present in all the chaotic windows, and the propensity for this type of motion increases with increasing frequency, as is evident in Figure 3. Figures 10 and 11 show the impact velocity spectra of the thin chaotic bands at frequencies of $0 \cdot 9$ and $0 \cdot 5$. Using the former as a reference, it can be seen that the number of grazing impacts also increases in the direction of decreasing frequency. 
Previous studies have concentrated on one impact motion, and these are evident in the major periodic regimes outside of the chaotic bands for frequencies larger than one. Inside the chaotic windows, and for the entire frequency range of less than one, there are motions with more than one impact. In particular, high $N$, low $P$ motion can be observed in several instances; for example, there are 5-3, 6-2 and 8-2 motions at frequencies of $2 \cdot 8,3 \cdot 25$ and 4.47, respectively, as illustrated in Figures 8 and 9.

The time histories and phase diagrams of representative $N-P$ motions for the mass $m_{1}$ are shown in Figures 12-14. For 1-4 motion, there is a high speed impact followed by a lower speed one and then grazing impacts. Then the decaying motion abruptly speeds up to initiate another cycle, showing traits of jerky movement. Grazing impacts are absent from the 1-2 motion, which also features one high speed collision. The 1-1 motion has a slightly lower impact speed, and this trend continues for the 2-1 and 3-1 motions. The mass $m_{1}$ undergoes secondary looping responses, as can be seen in Figure 14; for $N$ smaller than $P$ the secondary loops are close to the wall, resulting in more impacts, whereas for $N$ greater than $P$, the looping motion is away from the barrier, causing fewer impacts.

A picture on the transition to chaos at a frequency of about 1.8 is illustrated by phase diagrams and Poincaré sections in Figures 15 and 16. The period one motion undergoes a series of flip bifurcations to become chaotic.

A basin of attraction at a frequency of 2.755 is shown in Figure 17. The shaded areas correspond to 2-1 motion and the blank areas are for 17-10 motion. The initial displacement and velocity for $m_{2}$ are $-1 \cdot 0$ and $1 \cdot 0$, respectively. The basin is roughly divided into two halves, but there are scattered spots of 2-1 motion in the 17-10 region, and there is a substantial island of 17-10 motion in the 2-1 regime. It follows that in parts of the basin, small changes in initial conditions can lead to significant changes in motion quality. A basin of attraction for motion at frequency of $4 \cdot 25$ is shown in Figure 18. Periodic 12-8 motion exists in the shaded areas, whereas chaotic motion is present in the blank spaces. The overall pattern is largely irregular, as the two motions are mostly mixed

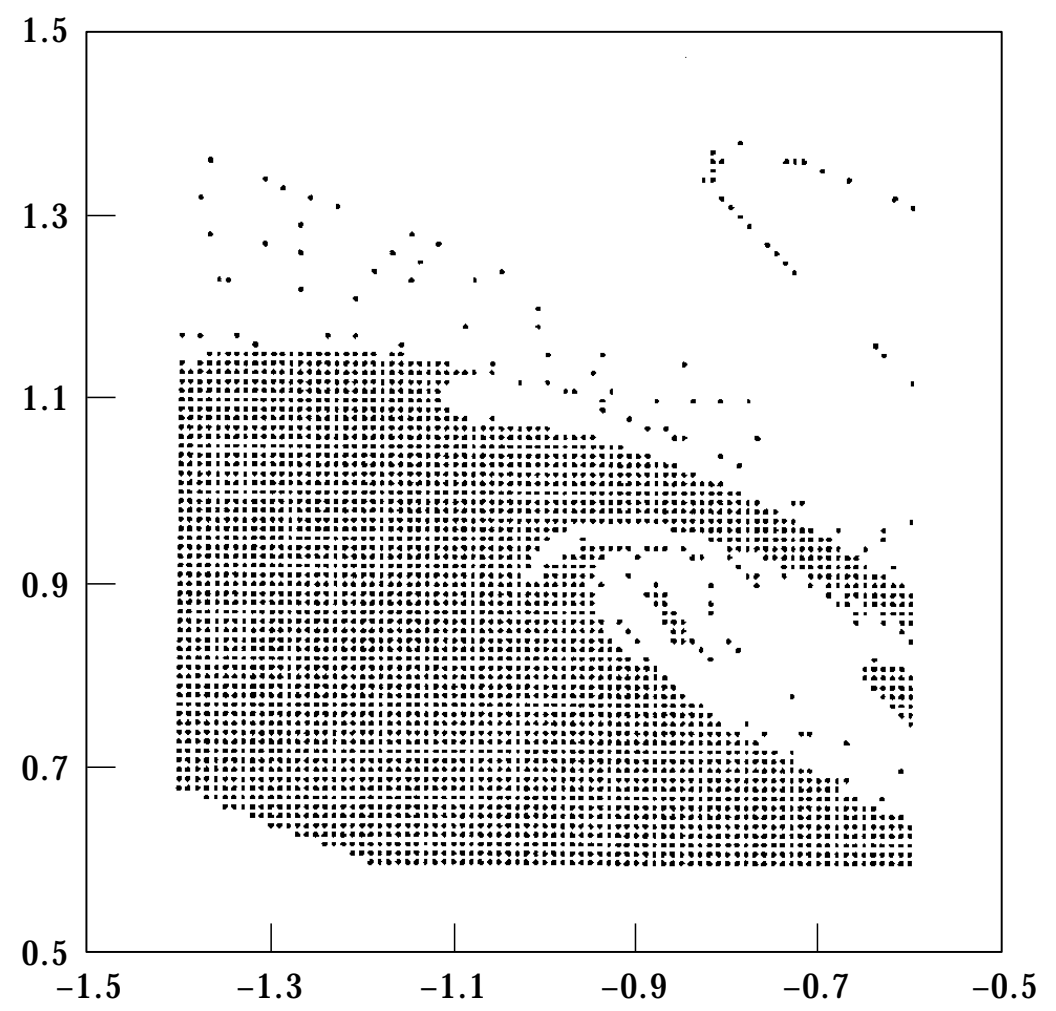

Figure 17. Basin of attraction for 2-1 (shaded), 17-10 (blank) motions, $\dot{x}_{1}(0)$ versus $x_{1}(0)$. 


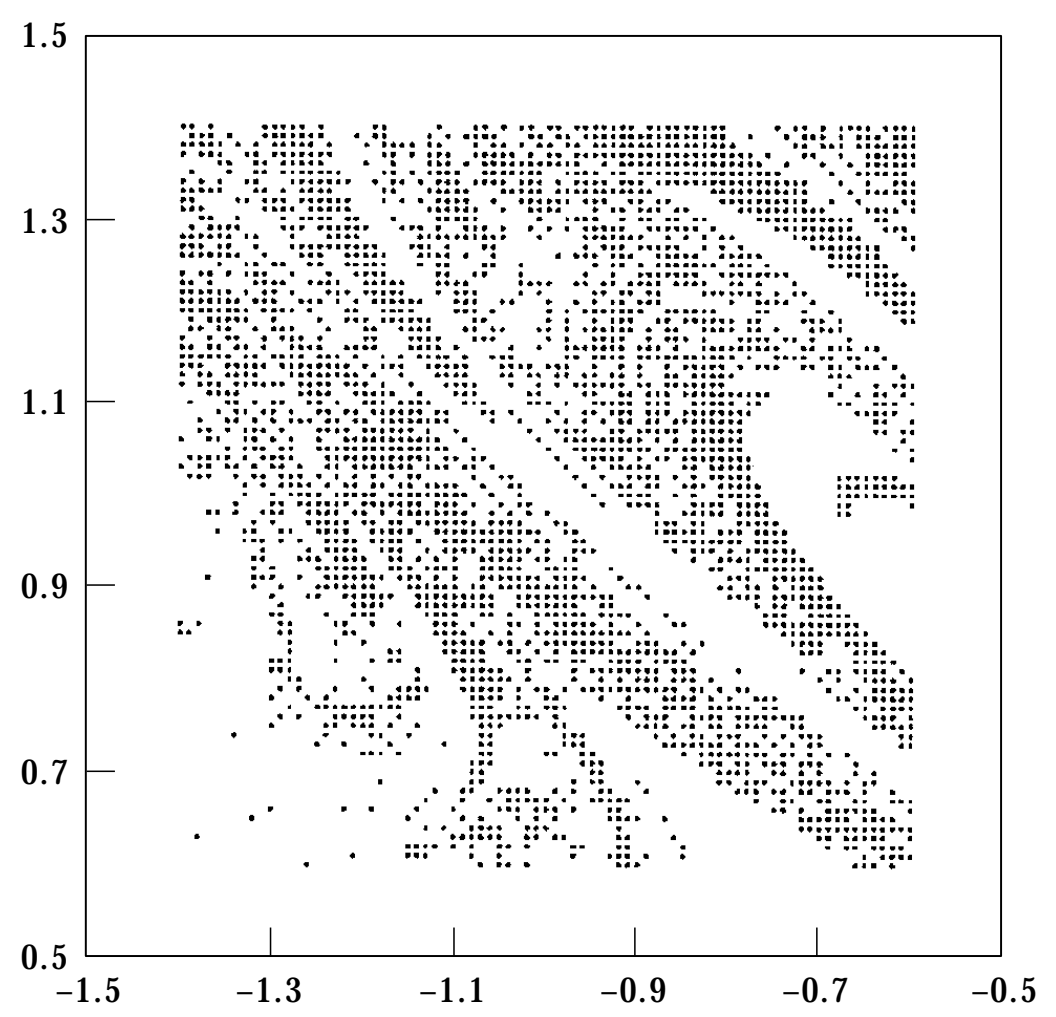

Figure 18. Basin of attraction for $12-8$ (shaded), chaotic (blank) motions, $\dot{x}_{1}(0)$ versus $x_{1}(0)$.

with no major distinctive boundaries. Clearly, small deviations in initial conditions can cause a periodic motion to shift to a chaotic one and vice versa.

\section{CONCLUSIONS}

The equations of motion for period $N$ motion with $P$ impacts for a multi-DOF impact vibrator subject to harmonic loadings were formulated. The variational equations for determining the stability of such motion were also obtained. The equations were applied to a two-DOF example, and different regimes of $N$-P response were obtained. Observations concerning the evolution of the dynamics with frequency were made, and in particular it is noted that, as the excitation frequency departs from the natural frequencies, grazing impacts become more numerous. High $N$, low $P$ motions were found to exist at the transition boundaries between harmonic and chaotic behaviour. Two basins of attraction were computed, and in one case it was shown that two very different types of motion can exist in a mixed pattern. The consequence is dynamics that is sensitive to initial conditions. This aspect was reinforced by the results of the second case, where periodic response is mingled with chaotic motion.

For frequencies larger than one, the major periodic windows contain period one or greater one impact motion. In contrast, for frequencies less than one, similar windows contain period one, one or more impacts motion. That such a wide range of dynamics exists is beneficial for applications, and one can infer from the displacement and velocity time histories useful characteristics for qualifying such motions from an engineering perspective. The 1-4 motion offers a high speed impact followed by hits of lower speed, the 1-1 motion gives high impact velocity and appears to be relatively smooth, whereas the 3-1 motion exhibits low impact velocity. 


\section{REFERENCES}

1. J. Shaw and S. W. Shaw 1989 Journal of Applied Mechanics, Transactions of the American Society of Mechanical Engineers 56, 168-174. The onset of chaos in a two-degree-of-freedom impacting system.

2. J. M. T. ThOMPSOn and R. Ghaffari 1982 Physics Letters 91A, 5-8. Chaos after period-doubling bifurcations in the resonance of an impact oscillator.

3. S. W. Shaw and P. J. Holmes 1983 Journal of Sound and Vibration 90, 129-155. A periodically forced piecewise linear oscillator.

4. N. Popplewell, C. N. Bapat and K. McLachlan 1983 Journal of Sound and Vibration 87, 41-59. Stable periodic vibroimpacts of an oscillator.

5. S. F. MASRI 1973 Journal of Applied Mechanics, Transactions of the American Society of Mechanical Engineers 40,127-132. Steady state response of a multidegree system with an impact damper.

6. J. O. AidanpaA and R. B. Gupta 1993 Journal of Sound and Vibration 165, 305-327. Periodic and chaotic behaviour of a threshold-limited two-degree-of-freedom system.

7. M. M. Nigm and A. A. Shabana 1983 Journal of Sound and Vibration 89, 541-557. Effect of an impact damper on a multi-degree of freedom system.

8. J. J. Moore et al. 1995 Journal of Vibration and Acoustics, Transactions of the American Society of Mechanical Engineers 117, 300-310. A forced response analysis and application of impact dampers to rotordynamic vibration suppression in a cryogenic environment.

9. D. Q. CAO and Z. Z. SHU 1997 Acta Mechanic Sinica 29, 74-83. Periodic motions and robust stability of the multi-degree-of-freedom systems with clearances. 